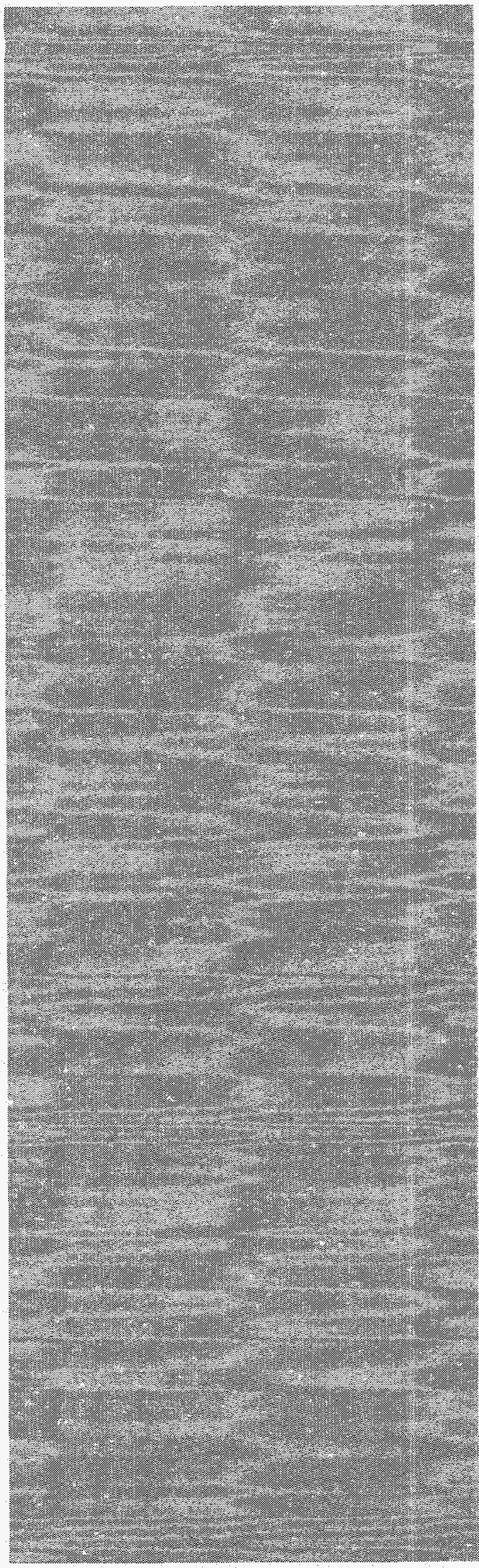

\title{
W-76 PBX 9501 Cylinder Tests
}

RECEIVED

\section{AUG 171998 \\ OSTI}

DISTRIBUTION OF THIS DOCUMENT IS UNUMTTED

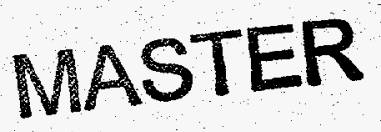

Los Alamos

NATIONAL LABORATORY

Los Alamos National Laboratory is operated by the University of California for the United States Department of Energy under contract W-7405-ENG-36. 
An Affirmative Action/Equal Opportunity Employer

This report was prepared as an account of work sponsored by an agency of the United States Government. Neither The Regents of the University of California, the United States

Government nor any agency thereof, nor any of their employees, makes any warranty, express or implied, or assumes any legal liability or responsibility for the accuracy, completeness, or usefulness of any information, apparatus, product, or process disclosed, or represents that its use would not infringe privately owned rights. Reference herein to any specific commercial product, process, or service by trade name, trademark, manufacturer, or otherwise, does not necessarily constitute or imply its endorsement, recommendation, or favoring by The Regents of the University of California, the United States Government, or any agency thereof. The viezos and opinions of authors expressed herein do not necessarily state or reflect those of The Regents of the University of California, the United States Government, or any agency thereof. Los Alamos National Laboratory strongly supports academic freedom and a researcher's right to publish; as an institution, however, the Laboratory does not endorse the viewpoint of a publication or guarantee its technical correctness. 


\section{DISCLAIMER}

Portions of this document may be illegible electronic image products. Images are produced from the best available original document. 
UC-741

Issued: July 1998

W-76 PBX 9501 Cylinder Tests

Larry G. Hill

Richard A. Catanach 


\section{CONTENTS}

ABSTRACT . . . . . . . . . . . . . . . . . . . 1

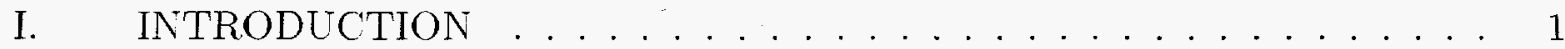

II. EXPERIMENTAL DETAILS . . . . . . . . . . . . . . 1

III. SAMPLE MATERIALS . . . . . . . . . . . . . . . 3

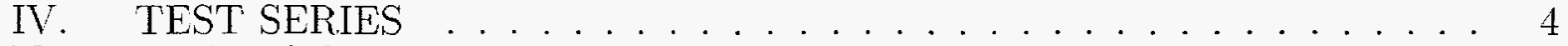

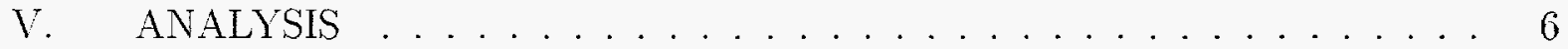

A. Fit to Wall Motion Data . . . . . . . . . . . . . . . 6

B. Cylinder Energy and its Determination ... . . . . . . . . 7

C. Gurney Energy and its Determination . . . . . . . . . . . . . 8

D. Determination of Detonation Velocity .............. . . 10

VI. RESULTS . . . . . . . . . . . . . . . . . . . . . . 11

A. Cylinder Energy . . . . . . . . . . . . . . . . . . . . . . 11

B. Gurney Energy . . . . . . . . . . . . . . . . . . . . . . . 12

C. Detonation Velocity . . . . . . . . . . . . . . . . . 13

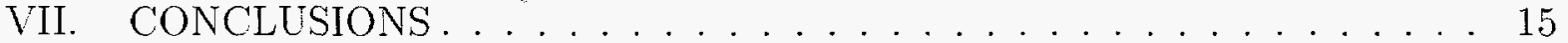

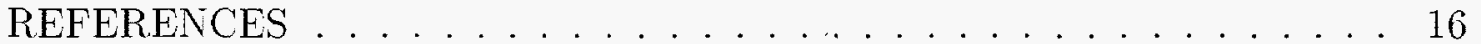

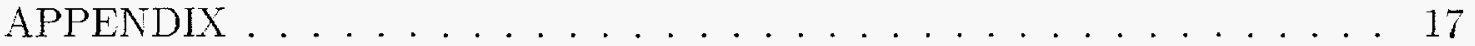

\section{LIST OF FIGURES}

1 Schematic diagram of the cylinder test. . . . . . . . . . . 3

2 Corrected wall expansion data $R-R_{0}$ vs. time, and the fit of Eqs. (1) and (2) (Shot \#15-2729). . . . . . . . . . . . . . . . . . . . . . . 7

3 Radial wall velocity (from differentiation of Eq. (1)) vs. wall expansion $R-R_{0}$ (Shot \#15-2729) . . . . . . . . . . . . . . . . . . 8

4 "Cylinder energy" vs. wall expansion $R-R_{0}$ (Shot \#15-2729) . . . . . . . . . 9

$5 \quad$ "Gurney energy" vs. wall expansion $R-R_{0}$ (Shot \#15-2729) . . . . . . . . . 10

$6 \quad x-t$ pin data and linear fit (Shot \#15-2729) . . . . . . . . . . . . 11

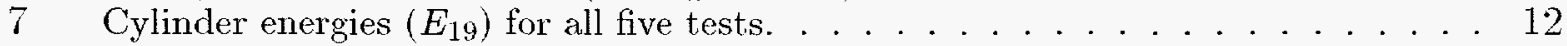

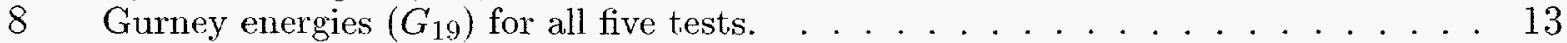

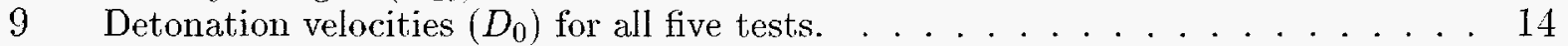

\section{LIST OF TABLES}

1 PBX 9501 Sample Information . . . . . . . . . . . . . . . . 4

2 Summary of Test Configurations . . . . . . . . . . . . . . . 6

3 Cylinder Energy Results . . . . . . . . . . . . . . . . . . . . . . . 12

4 Gurney Energy Results . . . . . . . . . . . . . . . . . . . . . . 14

5 Detonation Velocity Results . . . . . . . . . . . . . . . . 15 


\title{
W-76 PBX 9501 Cylinder Tests
}

by

\author{
Larry G. Hill and Richard A. Catanach
}

\begin{abstract}
Five 1-inch diameter cylinder tests were fired in support of the W-76 high explosive surveillance program. Three of the tests used baseline material, and two used stockpile return material. The diagnostics were electrical pins to measure detonation velocity and a streak camera to measure wall motion. The data was analyzed for cylinder energy, Gurney energy, and detonation velocity. The results of all three measures were consistent for all five tests, to within the experimental accuracy.
\end{abstract}

\section{INTRODUCTION}

Five cylinder shots were fired in support of the W-76 high explosive (HE) surveillance program. Three shots used a baseline lot of PBX 9501 (HOL89C730-010), meeting specifications for new material. Two shots used W-76 stockpile return material. This report describes the tests, analysis, and results. The project involved the efforts of a number of people, each of whom are acknowledged in the context of their contributions.

\section{EXPERIMENTAL DETAILS}

The shots were fired in essentially the standard manner, to be described in detail in a specification that is currently being written. Thus, only an overview of the test configuration is given here. Some small variations between experiments were explored in an attempt to clear up some minor long-standing issues. The results of these "sub-experiments" are discussed in some detail in Sec. 4.

Basically, the cylinder test consists of a copper tube filled with a solid or liquid explosive. The explosive is detonated at one end, and the motion of the tube is observed. While the 
test is simple in concept, much attention to detail is necessary to obtain high quality data. In particular, one must maintain tight mechanical tolerances and also pay close attention to the metallurgy of the copper.

The copper liners were made from oxygen-free high-conductivity (OFHC) copper, lnown officially as "Alloy 101." The tubes were 1 inch i.d. $\times 0.1$ inch wall $\times 11.8$ inches long. The tolerance on the i.d. and wall was \pm 0.0003 inch. The tubes were machined by Gerry Galusha (DX-5) at the TD Site shop. The i.d. along the axis was verified by an air-bore gauge. The tubes were annealed and etched under the direction of Carl Necker at MST-6. After being etched, the portion of the tube in the region of the slit was wound with plastic wrap to lieep the surface from oxidizing. The wrapping was removed just prior to firing the shot.

All the HE sample cylinders were machined about 1 mil under 1 inch diameter so as to barely fit into the copper tubes without interference. The baseline HE samples were 2 inches long, with 6 each per shot. The individual pieces were cored at group ESA-WMM and were finish-machined by George Harper (DX-5). The stockpile return HE cylinders were 1 inch long, with 12 each per shot. These pieces were cored from the weapon hemis and were finish-machined by George Harper.

The shots were assembled at DF Site by Bert Harry (DX-2). After the HE pieces were inserted in a tube, the stiack was gently compressed in a custom fixture (also designed by Bert Harry) until the stack lengtl was the sum of the individual segment lengths. The ends were then epoxied, and the assembly was held under load until the glue had fully cured. This process minimized the gaps between the individual HE segments, which cause small experimental errors and which tend to cut the tube prematurely. An SE-1 detonator, a 1/2-inch PBX 9407 booster pellet, and a P-16 plane wave lens were used to initiate the experiment.

Each tube had 10 electrical pins made of 2 mil enameled copper wires, to measure the detonation velocity. The wires were fastened to the tube surface with Kapton ${ }^{T M}$ tape, and their leading-edge location was precisely measured by a cathetometer. The tube itself acts as the circuit ground; the electrical circuit is completed when the wall movement caused by the detonation destroys the enamel insulation on the wire.

The detonation expands the tube into a funnel shape, as shown in Fig. 1. The radial expansion of the tube wall is observed at an axial location 7.5 inches from the initiator end of the tube, via a rotating mirror streak camera. This axial position is far enough from the initiating end so that the wave runs steady, yet far enough from the opposite end that the observed wall motion is unaffected by the tube's finite length. Also, at this location the 
slit is in the middle of an HE segment, and is thus as far as possible from joints between segments where the tube breaks first. The experiment is back-lit by an argon-bomb flash so that the experiment is viewed in silhouette. The flash is driven by a $1 \times 4 \times 4$ inch slab of Composition B. The shots were fired by Bert Harry and Don Murk (DX-4) at TA-40 Chamber 15. All tests were fired at the ambient outdoor temperature.

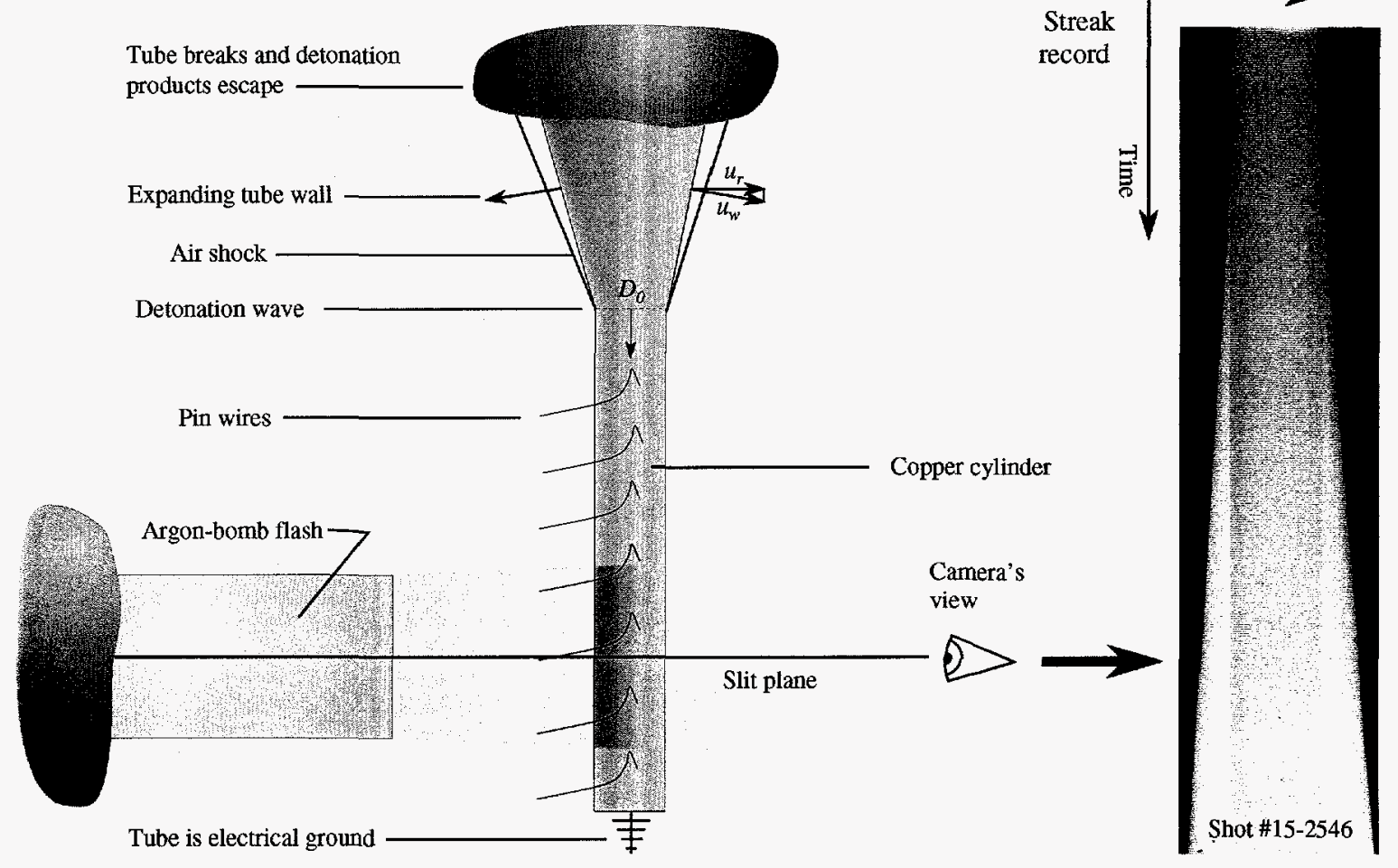

Figure 1. Schematic diagram of the cylinder test.

\section{SAMPLE MATERIALS}

All 18 pieces for the three baseline shots were cored from a single billet, which was hydrostatically pressed to weapons-specification density from PBX 9501 lot number HOL89C730010. (The ESA-WMM task order request number was P45852.) The density of five representative samples was measured by immersion by Jose Archuleta (DX-2), and the densities were found to be self-consistent at $1.832 \mathrm{~g} / \mathrm{cc}$. The baseline pieces were pressed at 20,000 psi and $85^{\circ} \mathrm{C}$, with 4 intensifications.

Both stockpile tests came from the same weapon, which was in the stockpile for 15 years. The HE cylinders for one stockpile shot were all taken from the forward hemi, and the HE cylinders for the other stockpile shot were taken from the aft hemi. The two hemis were 
Table 1. PBX 9501 Sample Information

\begin{tabular}{|c|c|c|c|c|c|c|}
\hline $\begin{array}{c}\text { Test } \\
\text { No. }\end{array}$ & $\begin{array}{c}\text { Shot } \\
\text { Number }\end{array}$ & $\begin{array}{c}\text { Material } \\
\text { Category }\end{array}$ & $\begin{array}{c}\text { Weapon } \\
\text { S/N }\end{array}$ & $\begin{array}{c}\text { Piece } \\
\text { Number }\end{array}$ & $\begin{array}{c}\text { PBX Lot } \\
\text { Number }\end{array}$ & $\begin{array}{c}\text { Density } \\
(\mathrm{g} / \mathrm{cc})\end{array}$ \\
\hline 1 & $15-2694$ & baseline 1 & NA & $45852-000(1 \rightarrow 6)$ & HOL89C730-010 & 1.832 \\
\hline 2 & $15-2695$ & baseline 2 & NA & $45852-000(7 \rightarrow 12)$ & HOL89C730-010 & 1.832 \\
\hline 3 & $15-2729$ & baseline 3 & NA & $45852-000(13 \rightarrow 18)$ & HOL89C730-010 & 1.832 \\
\hline 4 & $15-2730$ & stockpile 1 & 931065 & $76-204-01-21($ aft) & HOL77H685-004 & 1.835 \\
\hline 5 & $15-2731$ & stockpile 2 & 931065 & $76-103-09-33$ (fore) & HOL77H685-002 & 1.839 \\
\hline
\end{tabular}

manufactured from different lots of material, both of which were 20 years old. (Thus, the HE molding powder was evidently 5 years old when the weapon was constructed.) Additional information about the materials is given in Table 1.

\section{TEST SERIES}

The three baseline experiments were fired primarily to help establish a standard with which to compare future stockpile tests. At the same time, we wished to settle a few minor issues from the past, so as to converge on the best procedure for all future tests. One bit of lore is that powerful explosives such as PBX 9501 throw an oxide layer from the surface of the tube, which compromises the early part of the film record by throwing a "ghost" image ahead of the intended one. This problem has been traditionally dealt with by electropolishing or etching the surface of the tube. To examine this issue we fired the first control experiment with no surface treatment, and the second control experiment with an etching process performed by MST-6.

The ghost image was not seen on the first shot, but was seen on one side of the second shot (the one that was etched). One may conclude from this observation that the oxide explanation is likely not correct. It may be that it is actually an optical effect of the air shock that depends on the details of the light source, or that the tube spalls beneath the surface in a non-repeatable way. In any case, the uncertainty is only associated with the early expansion and should not affect the cylinder energy or Gurney energy measures, which are based on late expansion data. Even though the etching process did not appear to make a difference, we decided to have it done for every tube, since there is very little added labor (in addition to the annealing process) for MST-6 to do so.

The second issue is that the air shock refracts the light from the flash, perhaps enough so that the air shock may be confused with the tube surface early in the record ${ }^{1}$. For this 
reason, Wayne Campbell (GMX-8) reportedly fired cylinder shots in a helium atmosphere, since the refractive index of helium is smaller than that of air and does not increase with density nearly as much. We fired the third baseline shot in a helium atmosphere to see for ourselves what effect this had on the record. This test gave a cylinder energy about $1 \%$ higher than the average of the first two, which were within about $0.5 \%$ of each other. This suggested that refractive index effects might, in fact, be resolvable even late in the record.

However, there was another way in which the third baseline test differed from the first two. It had occurred to us that the magnification of the first two tests was a bit on the small side, and that by increasing it (since there was plenty of light) we could increase the resolution-seemingly for free. In retrospect we realized that this change rather than the helium - could account for the discrepancy, if the magnification of the lens system was not quite constant across the field.

Unfortunately we did not have another baseline shot to resolve this issue using identical materials, but having raised the issue, it was necessary to put the matter to rest. We therefore fired the first stockpile return shot at the larger magnification but in an air atmosphere, and found the resulting cylinder energy to be larger than the first two baseline shots (fired in air at the smaller magnification), and smaller than the third baseline shot (fired in . helium at the larger magnification). We then decided to fire the last stockpile shot at the larger magnification in a helium atmosphere to check the repeatability of that particular configuration. This last shot gave virtually the same cylinder energy as the previous shot (fired at the higher magnification in air).

Given the available information, there was no clear indication that the helium affected the measurement other than, perhaps, very early in the record. On the other hand, there did seem to be an effect of magnification since the three shots with the higher magnification all had cylinder energies higher than the two with the smaller magnifications. Suspecting this to be the case, we then characterized the magnification across the film plane by photographing a machinist's rule at various nominal magnifications. We found that there was indeed an aberration, which caused the magnification to change by about $1 \%$ between the center of the film and the edge (a distance that was somewhat larger than the half-width of the images). The application of this correction to the data resolved much of the discrepancy.

From these variations we concluded that (1) surface treatment of the tube has little effect, (2) helium had no clear effect and was not worth the trouble, (3) for best results a magnification correction should be made (at least for the model $72 \mathrm{~B}$ streal camera), and (4) one should pick an intermediate magnification and stick with it, as there is some consistency to be gained by performing each test identically. However, one should not confuse 
Table 2. Summary of Test Configurations

\begin{tabular}{|c|c|c|c|c|c|c|}
\hline $\begin{array}{c}\text { Test } \\
\text { Number }\end{array}$ & $\begin{array}{c}\text { Shot } \\
\text { Number }\end{array}$ & $\begin{array}{c}\text { Material } \\
\text { Category }\end{array}$ & $\begin{array}{c}\text { Tube } \\
\text { Finish }\end{array}$ & $\begin{array}{c}\text { Reference } \\
\text { Atmosphere }\end{array}$ & $\begin{array}{c}\text { Writing Speed } \\
(\mathrm{mm} / \mu \mathrm{s})\end{array}$ \\
\hline 1 & $15-2694$ & baseline 1 & as annealed & air & 0.2344 & 3.513 \\
\hline 2 & $15-2695$ & baseline 2 & etched & air & 0.2224 & 3.552 \\
\hline 3 & $15-2729$ & baseline 3 & etched & helium & 0.4722 & 3.530 \\
\hline 4 & $15-2730$ & stockpile 1 & etched & air & 0.4719 & 3.482 \\
\hline 5 & $15-2731$ & stockpile 2 & etched & helium & 0.4740 & 3.503 \\
\hline
\end{tabular}

consistency with absolute accuracy. For example, tests fired at the same magnification may be consistent with each other, but would all have a common systematic error to the extent that the magnification variation across the field is not accounted for.

Table 2 summarizes the various test configurations. The reference magnification is that based on the outer diameter of the tube, as that is what is measured from the film. The magnification elsewhere in the field is modulated by the calibration function found from optical tests, as mentioned above.

\section{ANALYSIS}

\section{A. Fit to Wall Motion Data}

Both sides of each film trace (corresponding to the left and right sides of the cylinder) were digitized on an optical comparator, 100 points from each side. When the data from the two sides are overlaid, the agreement is typically excellent. Nevertheless, one can usually improve the agreement a bit by making a correction, based on knowledge of the nature of the misalignment error. The two main types of misalignment error are the slit not being square to the shot and the slit not being square to the writing direction. Both types of error tend to cause time errors that are proportional to the radius.

The corrected data was fit using the empirical fitting form

$$
R(t)-R_{0}=\frac{\mathrm{v}_{\infty} t f(t)}{\frac{2 v_{\infty}}{a_{0}} f^{\prime}(0)+f(t)},
$$

where $f(t)$ is given by

$$
f(t)=(1+t)^{\omega}-1 .
$$

This form is a generalization of that suggested by Bill Davis ${ }^{2}$, and was developed primarily for equation-of-state analysis of the cylinder test ${ }^{3}$. In reality, the tube motion starts impulsively by shock loading, and it continues to "ring up" as waves reflect through it. The fit of 
Eqs. (1) and (2) smooths through the ringing, giving very nearly the trajectory that would be exhibited by an ideal incompressible wall. The fitting parameter $\mathrm{v}_{\infty}$ is the asymptotic wall velocity; $a_{0}$ is the initial acceleration (which is finite, consistent with the assumption of an incompressible tube), and $\omega$ is a fitting parameter. The fit is also allowed a virtual origin $t_{0}$ in the least-squares fitting process.

Figure 2 shows an example (baseline 3, shot \#15-2729) of corrected radius-time data with both sides overlaid, together with a nonlinear least-squares fit to the data using Eqs. (1) and (2). The fit residuals, magnified ten times for easy viewing, exhibit a symmetric damped oscillation indicating that the fit smooths through the ring-up in a near-optimum manner. This particular shot will be used as an example throughout this section, and the Mathematica file used to process it is given in the Appendix.

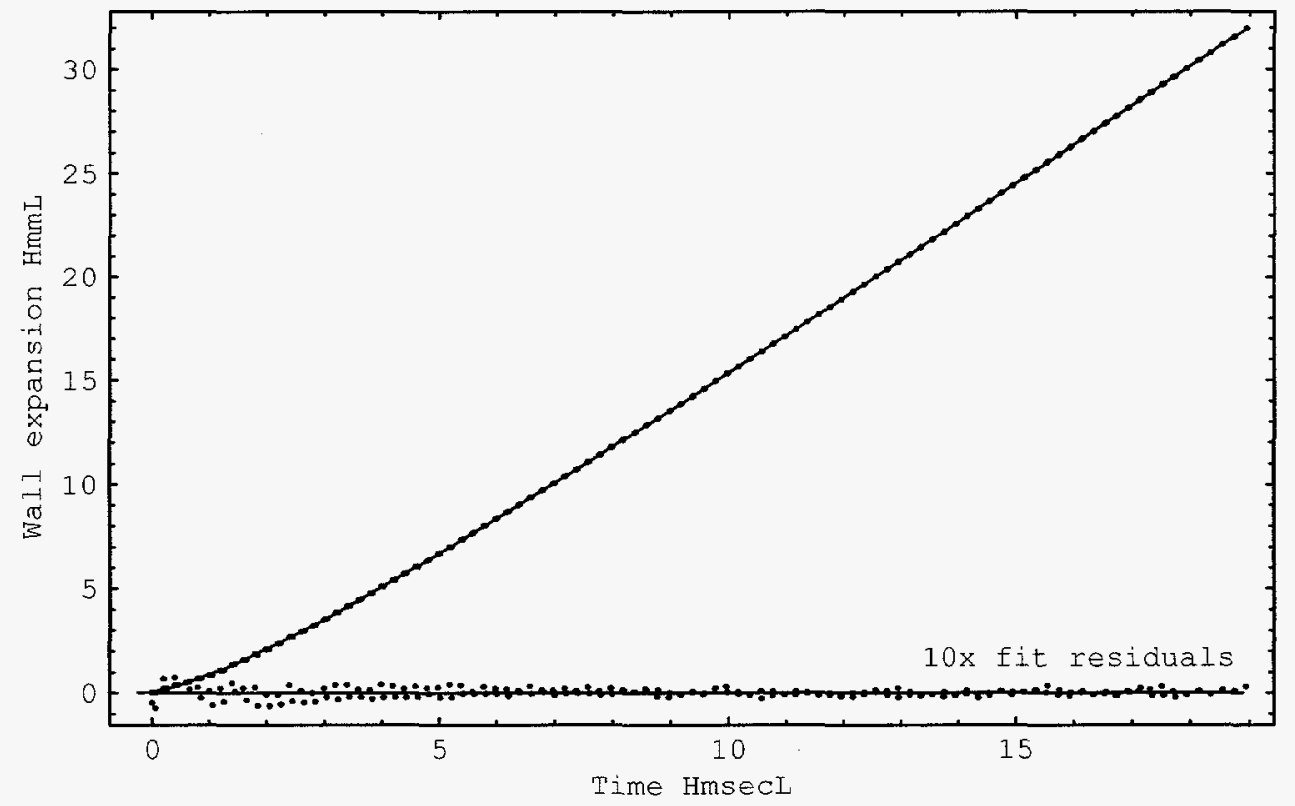

Figure 2. Corrected wall expansion data $R-R_{0}$ vs. time, and the fit of Eqs. (1) and (2) (Shot \#15-2729).

Equation 1 may be differentiated to give the radial component of wall velocity $v_{R}(t)$. One may plot this function parametrically versus the radial expansion $R(t)-R_{0}$ to show the radial velocity as a function of expansion. This curve, corresponding to the data and fit of Fig. 2, is shown in Fig. 3.

\section{B. Cylinder Energy and its Determination}

Half the radial velocity squared is close to the specific kinetic energy of the wall. (It is not exactly so because the mass motion has a small component of velocity in the forward 


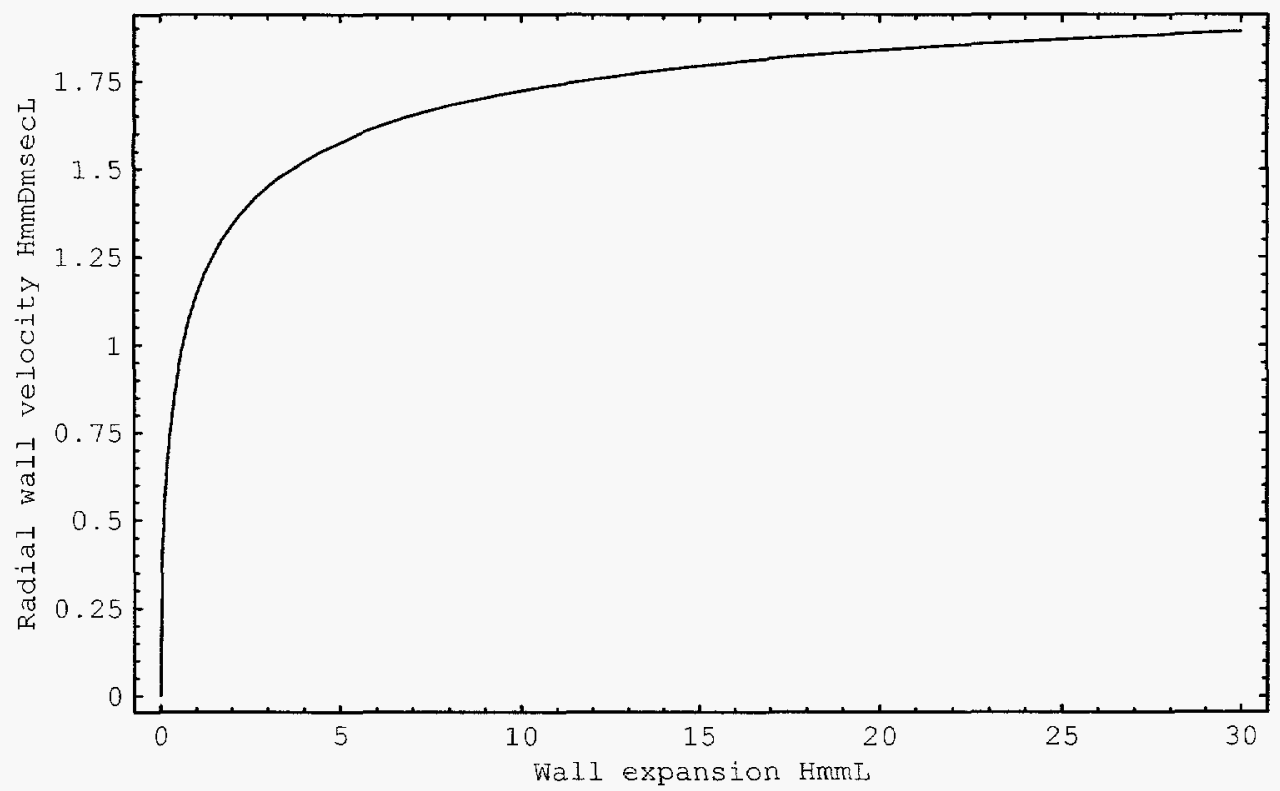

Figure 3. Radial wall velocity (from differentiation of Eq. (1)) vs. wall expansion $R-R_{0}$ (Shot \#15-2729).

direction.) This curve, also using the data and fit of Fig. 2, is shown in Fig. 4 as a function of the radial expansion. This quantity evaluated at 19 millimeters expansion is called the standard cylinder energy. It is the main historical figure of merit for the cylinder test and is denoted by $E_{19}$ :

$$
\left.E_{19} \equiv \frac{1}{2} v_{R}^{2}\right|_{R-R_{0}=19 m m} .
$$

Since the fitting form (Eqs. (1) and (2)) asymptotes to a constant velocity-and thus a constant specific energy - one may also determine the cylinder energy extrapolated to infinite expansion if desired.

\section{Gurney Energy and its Determination}

The Gurney energy is an approximation of the total kinetic energy of the explosive products and the surrounding tube, per unit mass of explosive. The Gurney calculation assumes that the tube expansion is cylindrical (rather than the true funnel shape), that the density of the detonation products is at all times uniform within the tube, that the tube has negligible strength, and that it stretches without breaking. The Gurney energy for cylindrical geometry is given by

$$
G\left(v_{R}\right)=\frac{1}{2} \mathrm{v}_{R}^{2}\left(\frac{1}{2}+\frac{m_{w / l}}{m_{H E / l}}\right)
$$




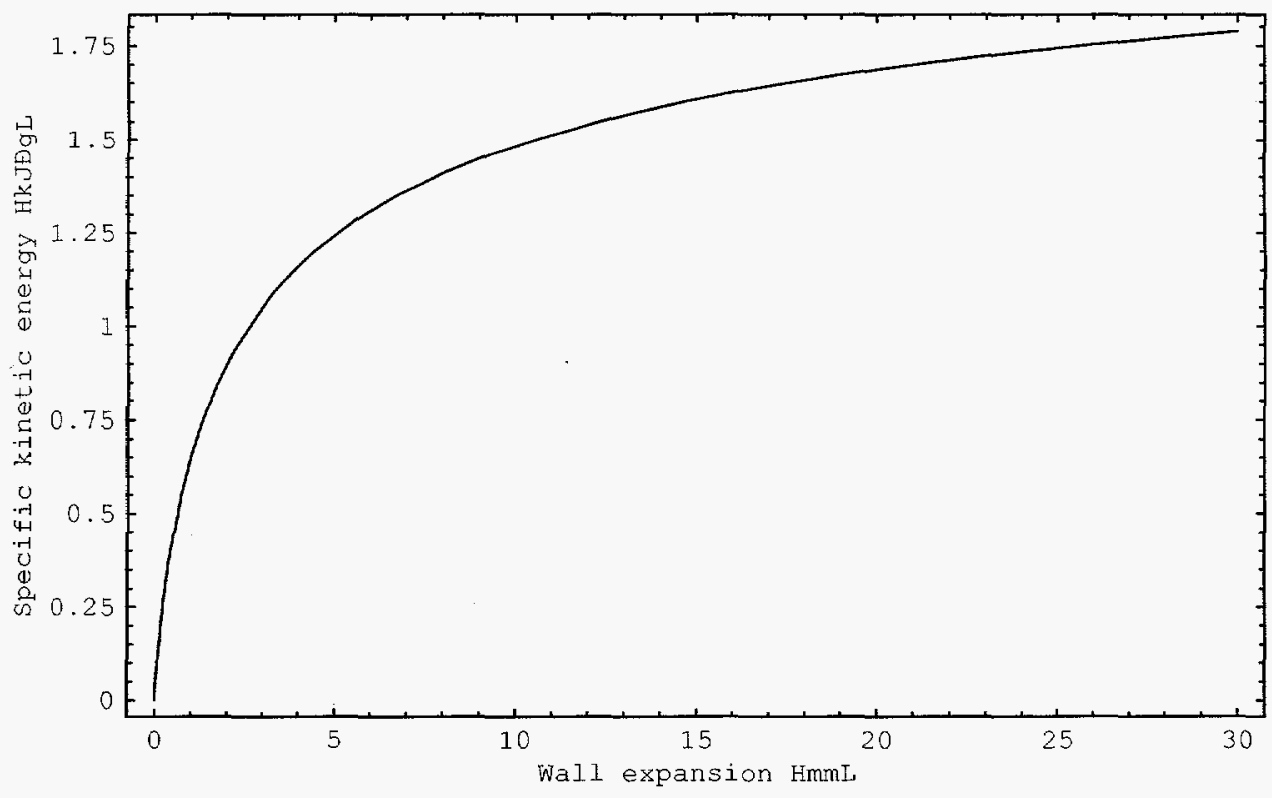

Figure 4. "Cylinder energy" vs. wall expansion $R-R_{0}$ (Shot \#15-2729).

where $m_{w / l}$ and $m_{H E / l}$ are the wall mass per unit tube length and the explosive mass per unit tube length, respectively.* Taking into consideration the geometry of the tube, the mass ratio can be alternatively written as

$$
\frac{m_{w / l}}{m_{H E / l}}=\frac{\rho_{w}}{\rho_{H E}}\left[\left(\frac{R_{0}}{r_{0}}\right)^{2}-1\right]
$$

where $\rho_{w}$ is the density of the wall, $\rho_{H E}$ is the initial density of the explosive, $R_{0}$ is the initial outer radius of the tube, and $r_{0}$ is the initial inner radius of the tube. The Gurney energy for the example case (shot \#15-2729) is plotted as a function of radial expansion in Fig. 5.

Typically, when the Gurney energy is quoted for a cylinder test it is, like the cylinder energy, evaluated at $19 \mathrm{~mm}$ radial expansion. This quantity will be denoted by $G_{19}$. Examination of Eq. (4) shows that $G_{19}$ is proportional to $E_{19}$ :

$$
\left.G_{19} \equiv G\left(\mathrm{v}_{R}\right)\right|_{R-R 0=19 m m}=E_{19}\left\{\frac{1}{2}+\frac{\rho_{w}}{\rho_{H E}}\left[\left(\frac{R_{0}}{r_{0}}\right)^{2}-1\right]\right\}
$$

As for the cylinder energy, one may determine the Gurney energy extrapolated to infinite expansion. This value, denoted by $G_{\infty}$, has special significance in that it is ideally the heat of detonation, assuming that all the chemical energy released during reaction is converted

${ }^{*}$ A good general discussion of Gurney calculations is given by Jones et al. ${ }^{4}$ 


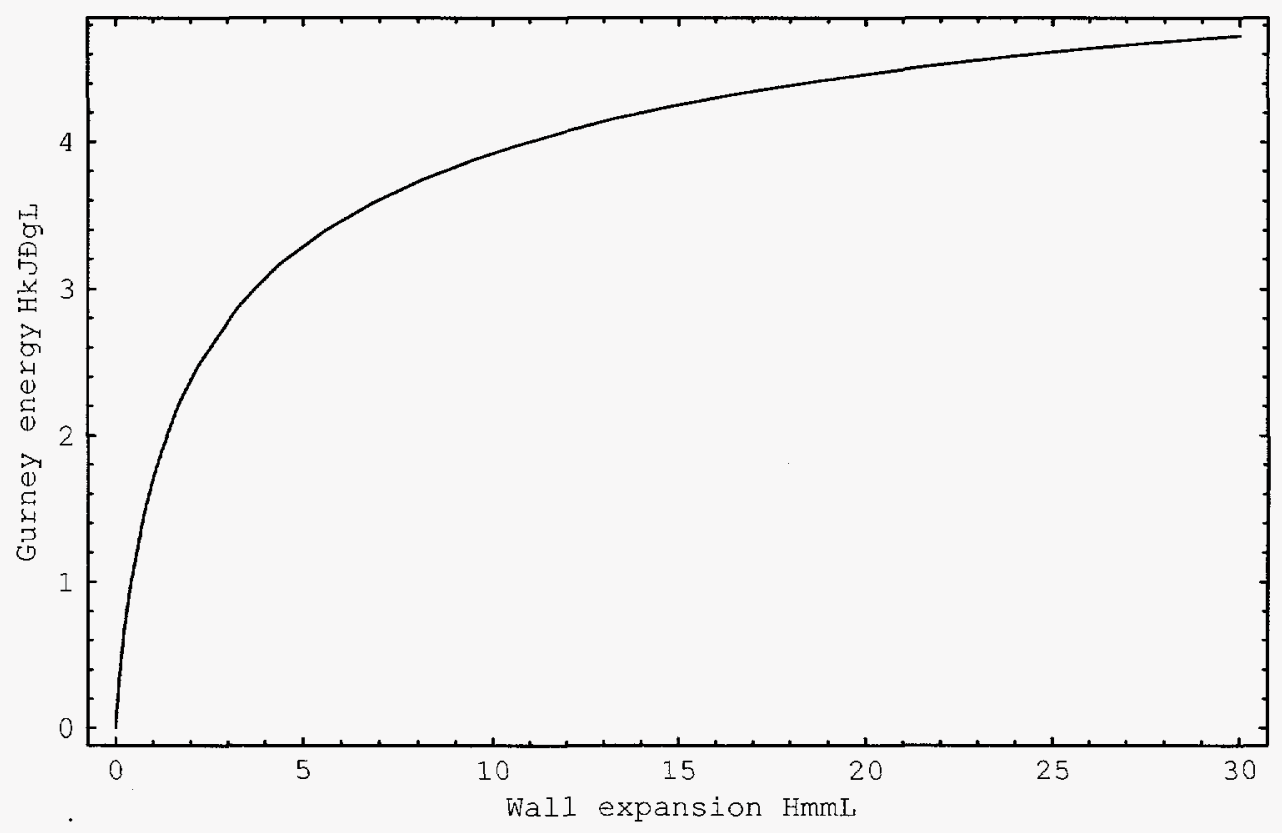

Figure 5. "Gurney energy" vs. wall expansion $R-R_{0}$ (Shot \#15-2729).

to mechanical energy in the way assumed by Gurney. In reality, there are various energy losses not accounted for, so that $G_{\infty}$ is in practice somewhat lower than the total chemical energy.

The density of the copper liner does not enter in the determination of the cylinder energy but does enter in the determination of the Gurney energy. The density value we used was the handbook value for alloy $101-8.94 \mathrm{~g} / \mathrm{cm}^{3}$ - as we did not have a means by which to measure it more accurately.

\section{Determination of Detonation Velocity}

To determine the detonation velocity one may plot the pin positions versus the pin times on an $x-t$ diagram as shown in Fig. 6 . The $x-t$ data are fit with a straight line, the slope of which is the detonation velocity. The uncertainty in detonation velocity associated with the random scatter in the data tends to be a few meters per second. In the case shown, the: standard deviation in velocity is $5.3 \mathrm{~m} / \mathrm{s}$. 


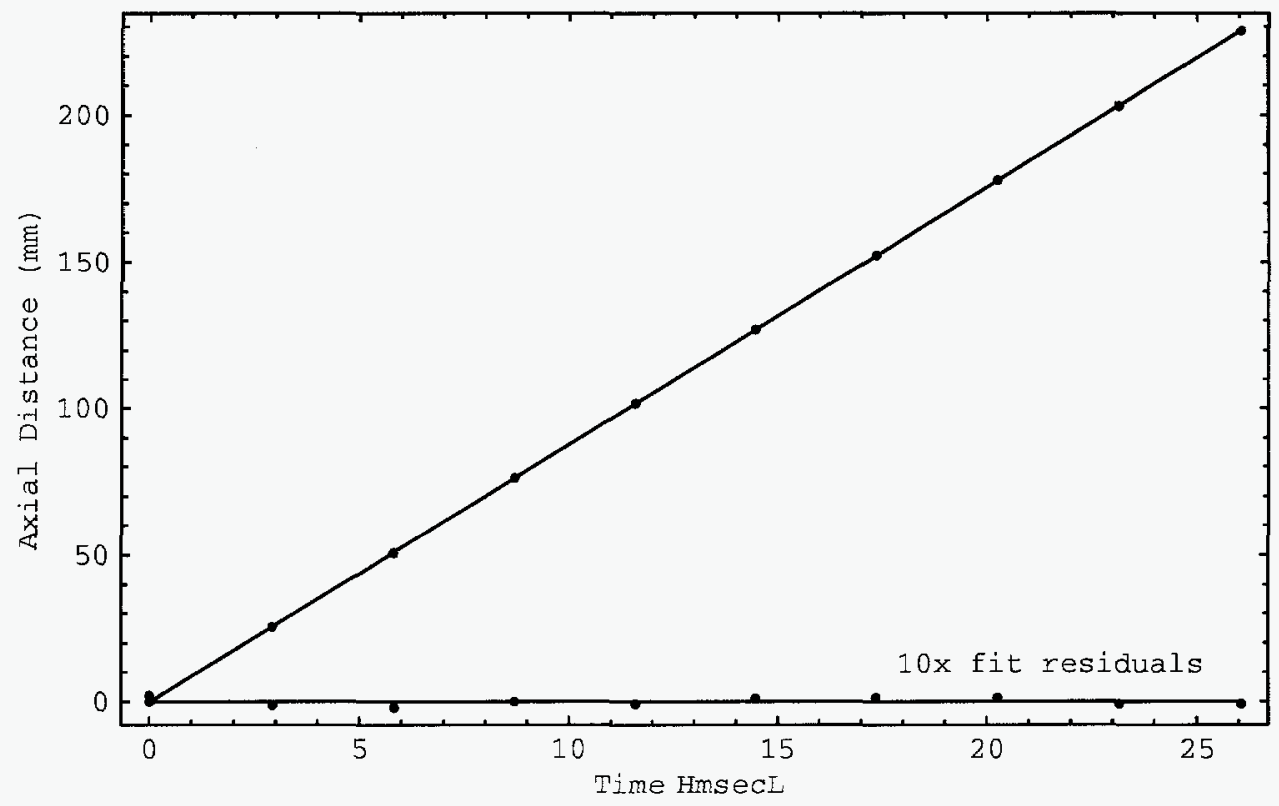

Figure 6. $x-t$ pin data and linear fit (Shot \#15-2729).

\section{RESULTS}

\section{A. Cylinder Energy}

The cylinder energies for all five tests are shown in Fig. 7. Taking all the data together, one finds a mean cylinder energy of $1.6601 \mathrm{~kJ} / \mathrm{g}$ and a standard deviation of $0.0097 \mathrm{~kJ} / \mathrm{g}$ (which is $0.58 \%$ of the mean). Another way to look at the data is to compare the average baseline value to the average stockpile value. Doing so gives a difference of $0.33 \%$. Either way, the variation between the baseline and stockpile samples is quite small.

One known source of variation between the five tests is that the stockpile samples are slightly denser than the baseline samples. This difference is easily estimated by making two seemingly reasonable assumptions: (1) for a given material and small changes in the initial pressed density, the nominal wall energy per unit tube length is proportional to the heat of detonation per unit tube length, and (2) the heat of detonation per unit mass of HE is constant for a given material. It is then straightforward to show that

$$
\frac{E}{E_{r e f}}=\frac{\rho}{\rho_{r e f}}
$$

where $E$ and $E_{r e f}$ are to be evaluated at the same expansion values. Given a measured value $E_{\text {ref }}$ corresponding to a certain pressed density $\rho_{r e f}, \mathrm{Eq} .(7)$ allows one to estimate what $E$ would be at some other pressed density $\rho$. In particular, let us normalize the stockpile samples to the density of the baseline samples, and recompute the mean and standard 


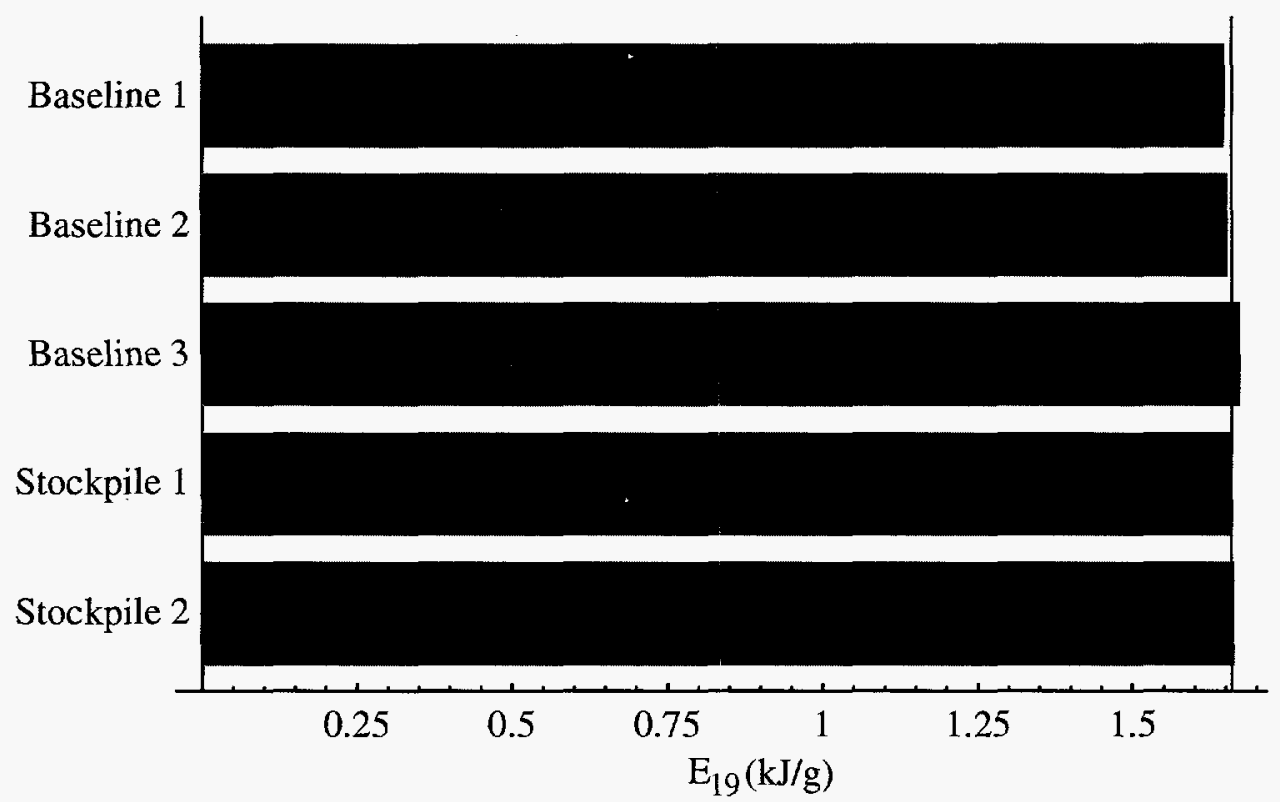

Figure 7. Cylinder energies $\left(E_{19}\right)$ for all five tests.

Table 3. Cylinder Energy Results

\begin{tabular}{|c|c|c|c|c|c|c|}
\hline $\begin{array}{c}\text { Shot } \\
\text { Number }\end{array}$ & $\begin{array}{c}\text { Material } \\
\text { Code }\end{array}$ & $\begin{array}{c}\text { Initial HE } \\
\text { Density } \\
\mathrm{g} / \mathrm{cm}^{3}\end{array}$ & $\begin{array}{c}E_{19} \\
\text { (unnorm.) } \\
\mathrm{kJ} / \mathrm{g}\end{array}$ & $\begin{array}{c}E_{\infty} \\
\left(\begin{array}{c}E_{19} \\
\mathrm{~kJ} / \mathrm{g}\end{array}\right.\end{array}$ & $\begin{array}{c}E_{19}(\text { normalized } \\
\left.\text { to } 1.832 \mathrm{~g} / \mathrm{cm}^{3}\right) \\
\mathrm{kJ} / \mathrm{g}\end{array}$ & $\begin{array}{c}E_{\infty}(\text { normalized } \\
\left.\text { to } 1.832 \mathrm{~g} / \mathrm{cm}^{3}\right) \\
\mathrm{kJ} / \mathrm{g}\end{array}$ \\
\hline $15-2694$ & baseline 1 & 1.832 & 1.6482 & 1.9426 & no change & no change \\
\hline $15-2695$ & baseline 2 & 1.832 & 1.6529 & 2.0457 & no change & no change \\
\hline $15-2729$ & baseline 3 & 1.832 & 1.6727 & 2.3523 & no change & no change \\
\hline $15-2730$ & stockpile 1 & 1.835 & 1.6623 & 2.5385 & 1.6596 & 2.5344 \\
\hline $15-2731$ & stockpile 2 & 1.839 & 1.6642 & 2.4573 & 1.6579 & 2.4479 \\
\hline
\end{tabular}

deviation. Taking all the data together, one now finds a mean cylinder energy of 1.6583 $\mathrm{kJ} / \mathrm{g}$ and a standard deviation of $0.0092 \mathrm{~kJ} / \mathrm{g}$ (which is $0.55 \%$ of the mean). This represents our best value at $1.832 \mathrm{~g} / \mathrm{cm}^{3}$. To obtain values at other densities, one may use Eq. (7). The difference between the average baseline and average stockpile cylinder energies is now $0.05 \%$. The agreement is somewhat improved, suggesting that the pressed density is, in fact, partially responsible for the small amount of data scatter.

The unnormalized and normalized numerical values for $E_{19}$ are shown in Table 3 . The unnormalized and normalized values of $E_{\infty}$ are also shown. These, being extrapolated quantities, have significantly more scatter than the $E_{19}$ values.

\section{B. Gurney Energy}

The Gurney energies for all five tests are shown in Fig. 8. Taking all the data together, one finds a mean Gurney energy of $4.3901 \mathrm{~kJ} / \mathrm{g}$ and a standard deviation of $0.0245 \mathrm{~kJ} / \mathrm{g}$ 
(which is $0.56 \%$ of the mean). Alternatively, the average baseline Gurney energy differs from the average stockpile value by $0.07 \%$. As for the cylinder energy, the variation between the baseline and stockpile samples is quite small in either case.

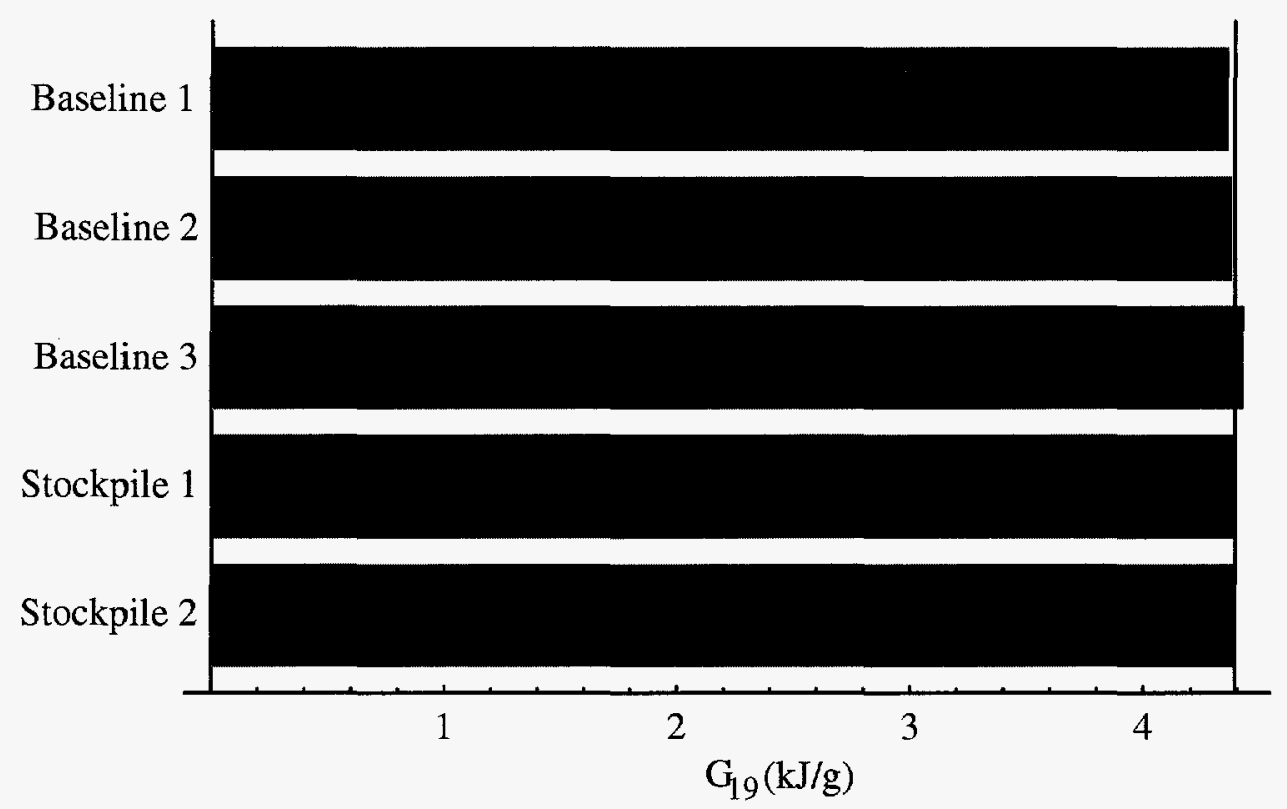

Figure 8. Gurney energies $\left(G_{19}\right)$ for all five tests.

One may correct for density differences in the Gurney energy as well, and for small deviations from the reference density one can show that the scaling law is the same as for the cylinder energy, i.e.,

$$
\frac{G}{G_{r e f}}=\frac{\rho}{\rho_{r e f}} .
$$

Performing this correction to all the data together, one now finds a mean Gurney energy of $4.3853 \mathrm{~kJ} / \mathrm{g}$ and a standard deviation of $0.0254 \mathrm{~kJ} / \mathrm{g}$ (which is $0.57 \%$ of the meanvirtually no change). This represents our best value at $1.832 \mathrm{~g} / \mathrm{cm}^{3}$. To obtain values at other densities, one may use Eq. (8). The difference between the average baseline and the average normalized stockpile Gurney energies is $0.20 \%$-slightly worse in this case than without density normalization.

The unnormalized and normalized numerical values for $G_{19}$ are shown in Table 4 . The unnormalized and normalized values of $G_{\infty}$ are also shown. These, being extrapolated quantities, have significantly more scatter than the $G_{19}$ values.

\section{Detonation Velocity}

The detonation velocities for all five tests are shown in Fig. 9. Taking all the data together, one finds a mean detonation velocity of $8.7959 \mathrm{~mm} / \mu$ s and a standard deviation 
Table 4. Gurney Energy Results

\begin{tabular}{|c|c|c|c|c|c|c|}
\hline $\begin{array}{c}\text { Shot } \\
\text { Number }\end{array}$ & $\begin{array}{c}\text { Material } \\
\text { Code }\end{array}$ & $\begin{array}{c}\text { Initial HE } \\
\text { Density }\end{array}$ & $\begin{array}{c}G_{19} \\
\text { (unnorm.) } \\
\mathrm{kJ} / \mathrm{g}\end{array}$ & $\begin{array}{c}G_{\infty} \\
\left(\begin{array}{c}\text { unnorm.) } \\
\mathrm{kJ} / \mathrm{g}\end{array}\right.\end{array}$ & $\begin{array}{c}G_{19} \text { (normalized } \\
\left.\text { to } 1.832 \mathrm{~g} / \mathrm{cm}^{3}\right) \\
\mathrm{kJ} / \mathrm{g}\end{array}$ & $\begin{array}{c}G_{\infty} \text { (normalized } \\
\left.\text { to } 1.832 \mathrm{~g} / \mathrm{cm}^{3}\right) \\
\mathrm{g} / \mathrm{cm}^{3} \mathrm{~kJ} / \mathrm{g}\end{array}$ \\
\hline $15-2694$ & baseline 1 & 1.832 & 4.3631 & 5.1423 & no change & no change \\
\hline $15-2695$ & baseline 2 & 1.832 & 4.3755 & 5.4152 & no change & no change \\
\hline $15-2729$ & baseline 3 & 1.832 & 4.4280 & 6.2270 & no change & no change \\
\hline $15-2730$ & stockpile 1 & 1.835 & 4.3946 & 6.7108 & 4.3874 & 6.6998 \\
\hline $15-2731$ & stockpile 2 & 1.839 & 4.3892 & 6.4847 & 4.3725 & 6.4600 \\
\hline
\end{tabular}

of $0.0161 \mathrm{~mm} / \mu \mathrm{s}$ (which is $0.24 \%$ of the mean). The average baseline detonation velocity differs from the average stockpile value by $0.18 \%$.

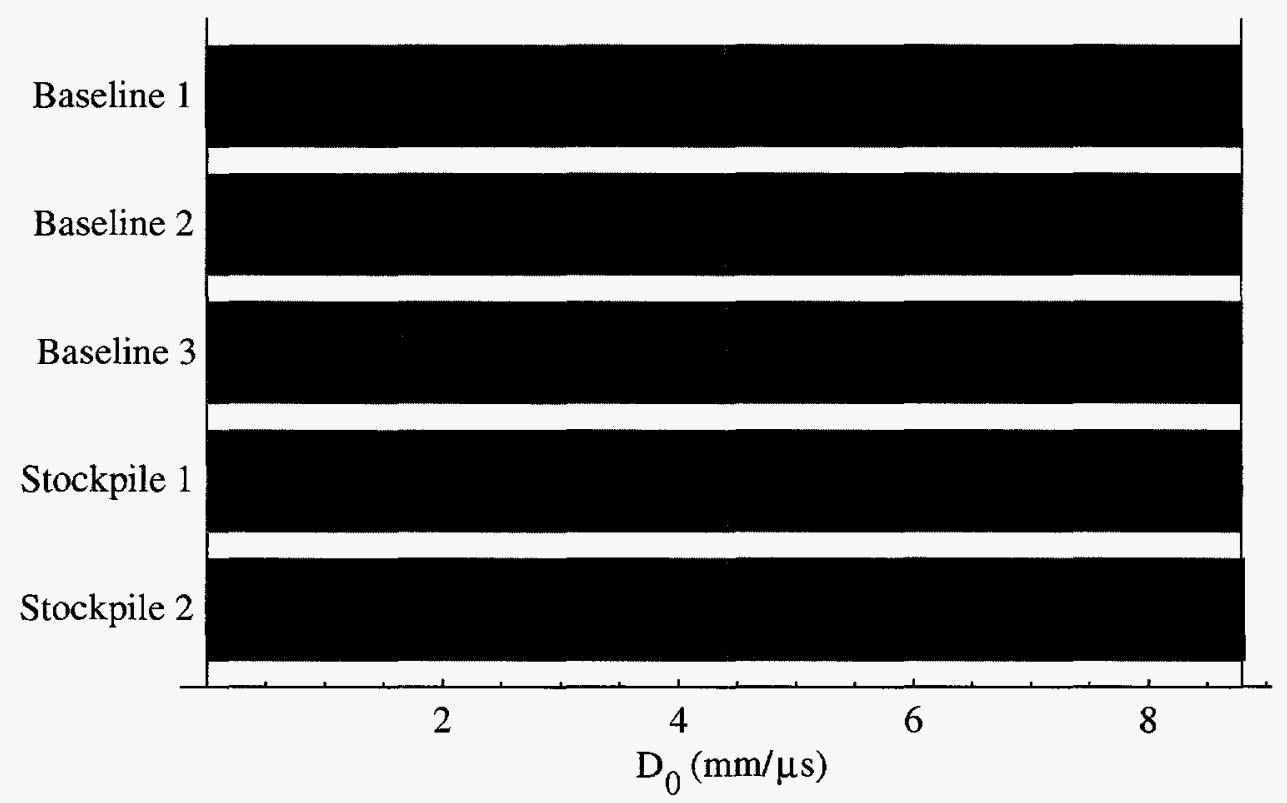

Figure 9. Detonation velocities $\left(D_{0}\right)$ for all five tests.

For solid explosives, it is observed that the detonation velocity increases linearly with density. Thus, the density correction is of the form

$$
D_{0}-D_{0, r e f}=\alpha\left(\rho-\rho_{\text {ref }}\right),
$$

where $\alpha$ is a material-dependant constant. The value used here is $3.76 \mathrm{~mm} / \mu \mathrm{s}$ per $\mathrm{g} / \mathrm{cc}$, which was determined by Catanach ${ }^{5}$ in a recent series of rate sticks having different densities. Applying this correction to all the data together, one now finds a mean detonation velocity of $8.7883 \mathrm{~mm} / \mu \mathrm{s}$ and a standard deviation of $0.0076 \mathrm{~mm} / \mu \mathrm{s}$ (which is $0.09 \%$ of the mean). This represents our best value at $1.832 \mathrm{~g} / \mathrm{cm}^{3}$. To obtain values at other densities, one may use Eq. (9). The difference between the average baseline and the average 
Table 5. Detonation Velocity Results

\begin{tabular}{|c|c|c|c|c|}
\hline $\begin{array}{c}\text { Shot } \\
\text { Number }\end{array}$ & $\begin{array}{c}\text { Material } \\
\text { Code }\end{array}$ & $\begin{array}{c}\text { Initial HE } \\
\text { Density } \\
\mathrm{g} / \mathrm{cm}^{3}\end{array}$ & $\begin{array}{c}D_{0} \\
\text { (unnormalized) } \\
\mathrm{mm} / \mu \mathrm{s}\end{array}$ & $\begin{array}{c}D_{0} \text { (normalized } \\
\text { to } 1.832 \mathrm{~g} / \mathrm{cm}^{3} \text { ) } \\
\mathrm{mm} / \mu \mathrm{s}\end{array}$ \\
\hline $15-2694$ & baseline 1 & 1.832 & 8.7816 & no change \\
\hline $15-2695$ & baseline 2 & 1.832 & 8.7981 & no change \\
\hline $15-2729$ & baseline 3 & 1.832 & 8.7829 & no change \\
\hline $15-2730$ & stockpile 1 & 1.835 & 8.7952 & 8.7839 \\
\hline $15-2731$ & stockpile 2 & 1.839 & 8.8215 & 8.7952 \\
\hline
\end{tabular}

normalized stockpile detonation velocities is $0.02 \%$. The agreement is somewhat improved, suggesting that the pressed density is, in fact, partially responsible for the small amount of data scatter. The unnormalized and normalized numerical values for $D_{0}$ are shown in Table 5 .

\section{CONCLUSIONS}

The results for cylinder energy, Gurney energy, and detonation velocity are consistent for all five tests to within what is believed to be the accuracy of the experiments. The stockpile return samples had slightly higher densities than the baseline samples, and this fact appeared to account for a fraction of the (already small) relative deviation between the five tests. This conclusion was reached by making reasonable corrections for these density differences and noting that the agreement was generally, though not universally, improved as a consequence.

There were a few intentional differences between the tests which were intended to help determine a final standard for future tests. The factors explored were the surface treatment of the tube, the atmosphere around the tube (air versus helium), and the magnification. Only the latter affected the results in a noticeable way, due to a small aberration in the optical system which caused the magnification to vary across the field. This aberration was characterized and then applied as a correction in the data analysis. 


\section{REFERENCES}

1. W. C. Davis, "Cylinder Test-Light Refraction in the Air Shock," Los Alamos Group M-3 (Internal) Quarterly Report M-3-MR-73-4, pp. 46-52 (1973).

2. W. C. Davis, "Cylinder Test Shot," Los Alamos Group M-9 (Internal) Quarterly Report M-9-QR-87-3, pp. 28-32 (1987).

3. L. G. Hill, "Detonation Product Equation-of-State Directly from the Cylinder Test," Proceedings 21st Int. Symp. on Shock Waves, Great Keppel Island, AUSTRALIA (1997).

4. G. E. Jones, J. E. Kennedy, and L. D. Bertholf, "Ballistics Calculations of R. W. Gurney," Am. J. Phys. 48(4), pp. 264-269 (1980).

5. R. A. Catanach, "Variation in PBX 9501 Detonation Velocity with Density," Los Alamos National Laboratory Memorandum DX-1-98-036 to Bart Olinger, ESA-WMM (1998). 


\title{
Appendix
}

\section{Mathematica Analysis of Cylinder Test Shot \#15-2729}

\author{
Version: 1.3 \\ Date: $5 / 1 / 98$ \\ By: Larry Hill, Group DX-1, LANL
}

\section{Notebook and Analysis Information (Read this first.)}

This program operates interactively. The user is prompted for information and must make decisions based on intermediate results. The commands should be executed in sequence. The cells that are highlighted with a box require the user to supply information before executing the command.

The program assumes that the cylinder expansion data is read in a particular way. The film is aligned with the writing direction horizontal, with time increasing to the right. The spatial film coordinate $x$ is read as a function of the time film coordinate $y$. The units on the optical comparator should be set to millimeters. Both sides of the data trace are read into separate files. The time origin is set to the "jump-off" point, where the tube expansion begins. The time origin may be set seperately for each side, or it may be left the same for each side. (The two time origins will be mutually adjusted for optimum agreement.)

It is important not to remove or adjust the film between the top and bottom readings, and the two sides should be read with little delay in between so as to minimize dimensional changes due to shifts in ambient temperature.. As read, the upper trace " $U$ " will have positive $x$-values and the lower trace " $L$ " will have negative $x$-values.

The pin data is entered manually in Section 10 , according to the instructions there.

Date and version information:

Date[]

$\{1998,5,4,13,44,25\}$

\$version

NeXT 3.0 (July 11, 1997)

This command turns off spelling error warning messages:

Off [General : :spel11] 


\section{Shot Information}

Shot number:

$15-2729$

Shot date:

9/23/97

Description:

Reference shot for surveillance. Fired in helium atmosphere.

Temperature: Ambient

Shot parameters:

Specify the reference magnification, corresponding to the tube o.d.:

$$
\text { magRef }=0.4722
$$

0.4722

Specify the writing speed (assumed to be constant across the film):

$$
\text { wspeed }=3.530(* \mathrm{~mm} / \mu \mathrm{sec} *)
$$

3.53

Specify the outer radius of the tube:

$\mathrm{RO}=15.240(* \mathrm{~mm} *)$

15.24

Specify the inner radius of tube:

ro $12.700(* \mathrm{~mm} *)$

12.7

Specify the density of the tube:

$$
\rho w=8.94(* g / c c *)
$$

8.94

Specify the initial HE density: 
$\rho 0=1.832(* \mathrm{~g} / \mathrm{Cc} *)$

1.832

\section{Film Data}

This command sets the directory to where the data files reside. Specify the path:

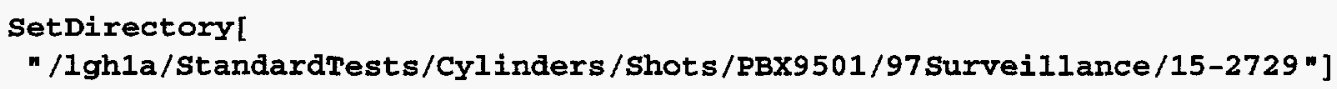

/lghla/StandardTests/Cylinders/Shots/PBX9501/97Surveillance/15-2729

These two commands read the data files. Files are identified by $U$ for "upper" and L for "lower," but it is arbitrary which is which. Recall that $x$ is the spatial film coordinate and $y$ is the temporal film coordinate. Specify the file names:

dataUyx = ReadList ["15-2729U.1gh" , \{Real, Real \}];

dataLyx = ReadList ["15-2729L.1gh", \{Real, Real\}];

\section{Conversion to Real Space-Time}

This is the mapping to real space-time coordinates. Here $t$ denotes time and $e=R-R 0$ denotes the radial expansion of the outer tube surface relative to its initial position. The term $R$ is used to denote the outer radius, and $r$ is used to denote the inner radius.

The magnification will generally vary slightly across the width of a streak camera. The following formula is a correction for the Los Alamos model $72 \mathrm{~B}$ camera and a particular lens (Rodenstock $800 \mathrm{~mm}$ ). One may substitute different corrections, or assume a constant magnification, as appropriate. This equation describes the measured magnification, normalized by the centerline value, as a function of the spatial film coordinate $\mathrm{x}$ measured from the center of the film:

$$
\begin{aligned}
& \operatorname{MF} 800\left[x_{-}\right]=1+\frac{9.6642 x^{2}}{10^{6}} \\
& 1+9.6642 \times 10^{-6} x^{2}
\end{aligned}
$$

This is the actual magnification, which makes use of the reference magnification magRef measured at an $x$ position RO*magRef:

$$
\begin{aligned}
& \operatorname{mag}\left[\mathbf{x}_{-}\right]=\frac{\text { magRef MF800 }[\mathbf{x}]}{\text { MF800 [R0 magRef }]} \\
& 0.471964\left(1+9.6642 \times 10^{-6} \mathrm{x}^{2}\right)
\end{aligned}
$$


This is the mapping function to real time and space coordinates:

$$
\text { FNte[w_] }:=\left\{\frac{\mathrm{w}[1 \rrbracket}{\text { wspeed }}, \frac{\mathrm{Abs}[\mathrm{w} \llbracket 2 \rrbracket]}{\operatorname{mag}[\mathrm{w}[2 \mathbb{1}]}\right\}
$$

Apply the mapping to the data files:

$$
\begin{aligned}
& \text { dataute }=\text { Map }[\text { FNte }, \text { dataUyx }] ; \\
& \text { datalte }=\text { Map }[\text { FNte, dataLyx]; }
\end{aligned}
$$

This is the uncorrected combined data set:

$$
\text { dataulte = dataute U datalte; }
$$

This is a plot of the uncorrected combined data set. Specify the shot number for the title:
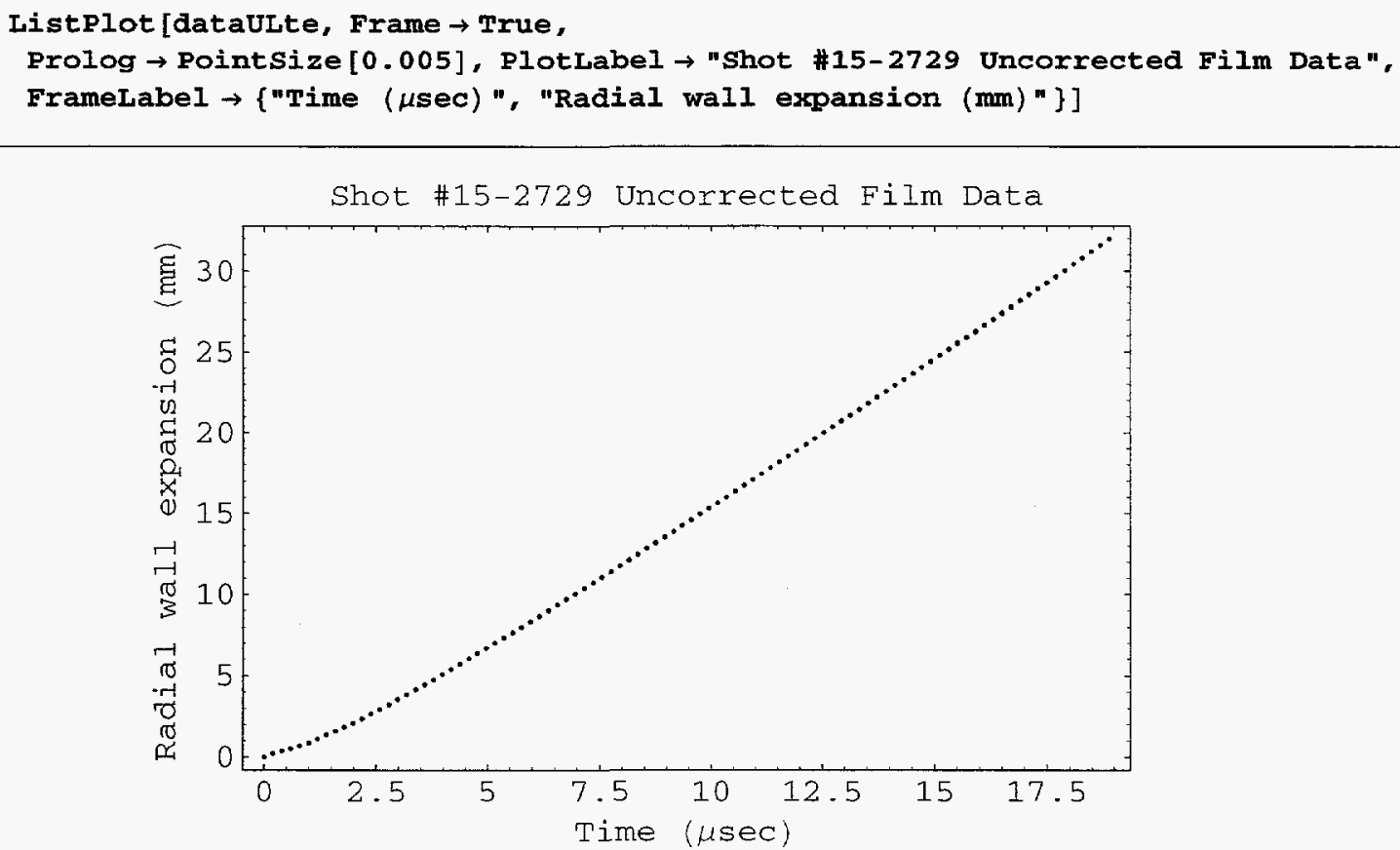

\section{Tilt and Time-Shift Correction}

The most prevalent alignment errors are (1) the slit not being perfectly normal to the writing direction, and (2) the slit not being perfectly square with the shot. Both of these errors cause time errors proportional to the radius. For most shots such errors are greater (though often only slightly so) than the background noise. One may remove this error by comparing both sides of the record, and using the fact that the error is anti-symmetric about $R=0$.

It is equivalent, and simpler, to work with the time as a function of expansion, $t[e]$, rather than of radius. This just introduces a relative shift in the time origins, which is readily accounted for. These commands transpose the data:

FNet $\left[w_{-}\right]:=\{w \llbracket 2 \rrbracket, w \llbracket 1 \rrbracket\}$ 
dataUet $=$ Map $[$ FNet, dataute $]$;

dataLet $=$ Map [FNet, dataLte $]$;

We now perform an interpolated fit to the data in order to subtract the two data sets:

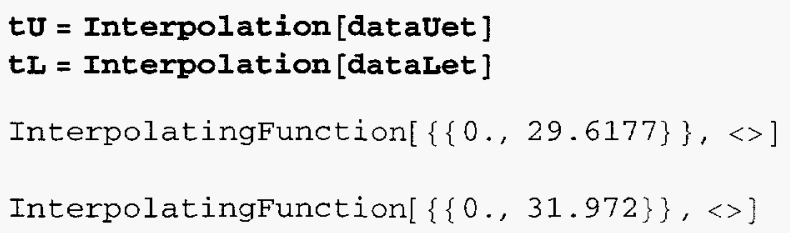

Find maximum time and radius values for plotting. Tmax is the maximum time of the combined data set:

Tmax $=$ Max $[$ Thread $[$ datauLte $][1 \rrbracket]$

18.8924

The variable emaxlst is the maximum expansion of the side that breaks first:

emaxist $=\operatorname{Min}[\operatorname{Max}[$ Thread $[$ dataUte $] \llbracket 2 \rrbracket], \operatorname{Max}[$ Thread [dataLte] [2】]]

29.6177

The variable emax 2 nd is the maximum expansion of the side that breaks second:

emax2nd $=$ Max $[$ Thread $[$ datauLte] [12]]

31.972

This is a plot of the two interpolation functions for time as a function of expansion. Specify the shot number for the title:

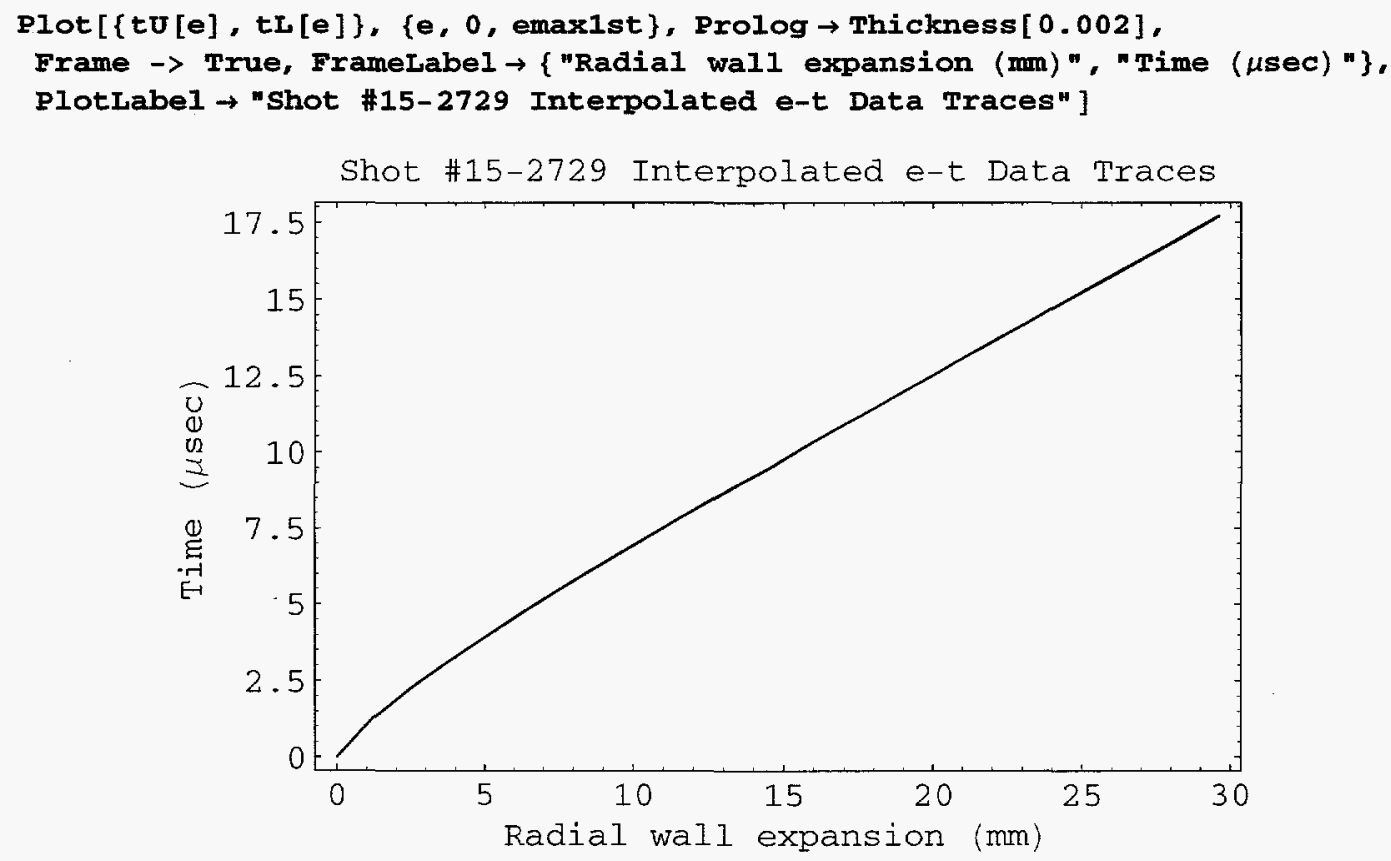


This is the time difference between the two traces as a function of radial wall expansion:

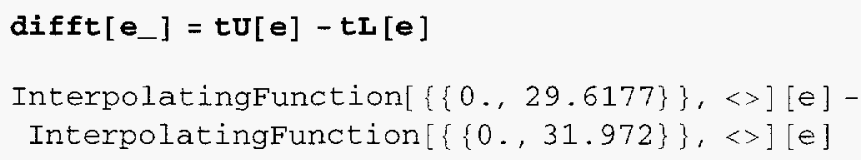

This is a plot of the difference between the two above interpolation functions. Specify the shot number for the title:

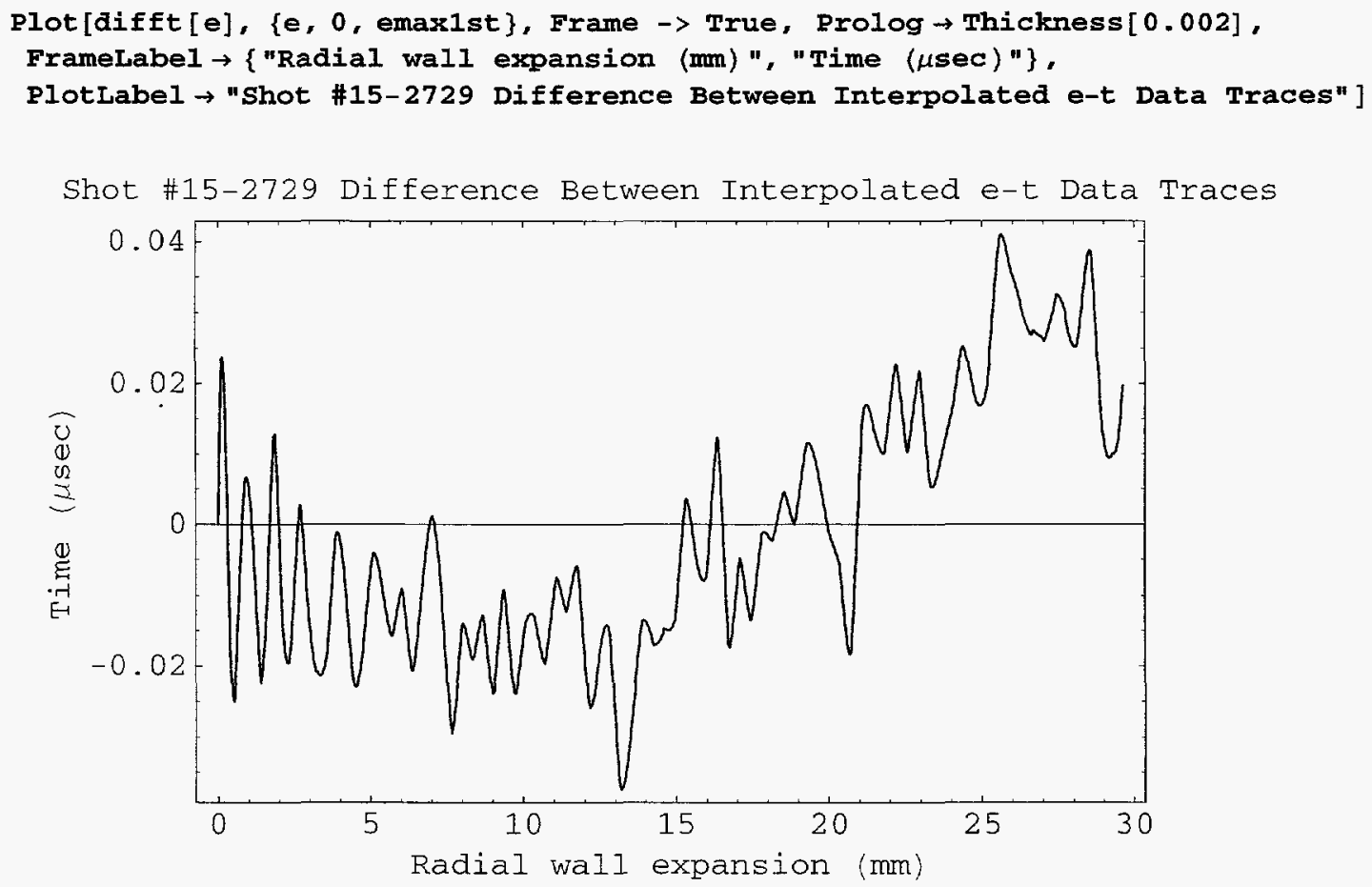

The premise is that the above difference will be basically linear (with quite a bit of noise superimposed). The intercept is the relative error between the two time origins, and the slope is the relative tilt. Often the data near the origin (which tends to be a bit erratic) will deviate from the overall linear trend and will need to be pruned, so as to best fit the linear portion of the curve. You will need to look at the curve and decide which portion (if any) to cut off. Specify echop, the wall expansion below which the fitting data will be dropped:

echop $=13$

13.

The following commands generate a pruned list of points from the above difference function, and fit a line to it:

$\operatorname{dataDiff}=$ Table $[\{e, \operatorname{difft}[e]\},\{e$, echop, emax1st, 0.1 $\}]$;

Delete the following intermediate plot after evaluation:

plot1 = ListPlot [atadiff] 
This command performs a linear fit to dataDiff:

$$
\begin{aligned}
& \text { fitDifft [e_] = Fit [dataDiff, }\{1, e\}, e] \\
& -0.0591224+0.00314457 \mathrm{e}
\end{aligned}
$$

Delete the following intermediate plot after evaluation:

$$
\text { plot2 }=\text { plot }[\text { fitDifft }[e],\{e, 0, \operatorname{emax} 2 n d\}]
$$

This is a plot of the time difference data between $e-t$ records, and the linear fit to it. Specify the shot number for the title:

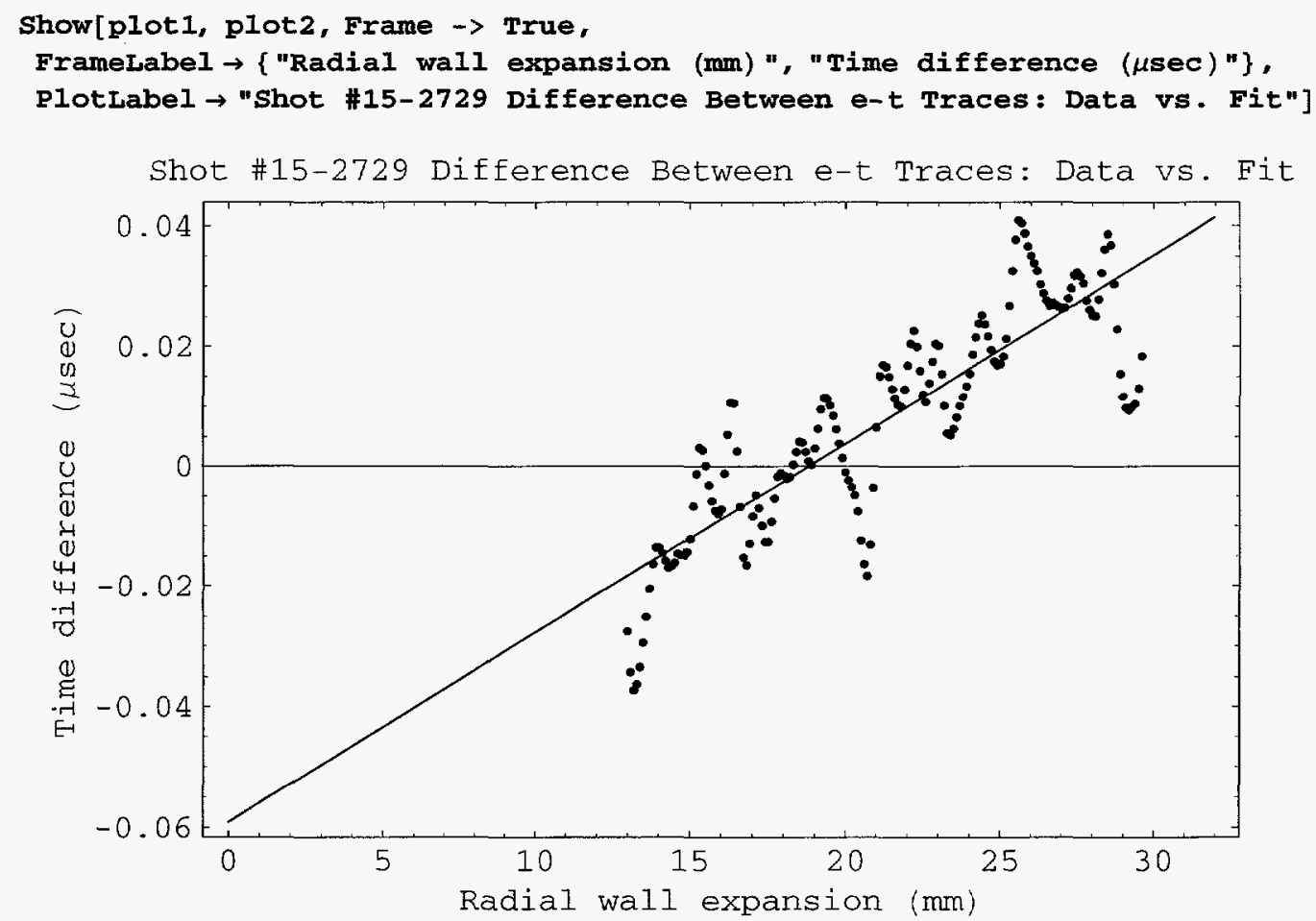

The following commands apply the correction, determined by the above fit, to the data points. The data is also transposed back to e as a function of $t$ at the same time.

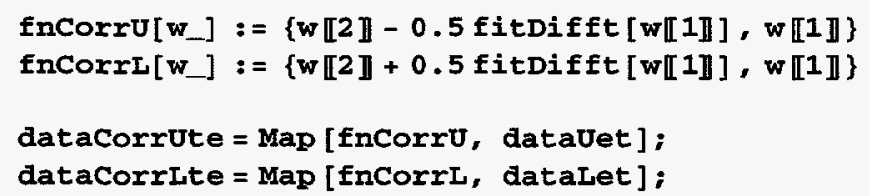

The combined corrected data set is:

datacorrte $=$ datacorrute U datacorrLte;

The time origin shift just performed will generally give a point or two with negative time values. It is desirable to shift the combined set to make the first data point correspond to zero time; otherwise, the fitting form will be complex in that region. The following command shifts the time origin so that the first value is zero: 
fnshift $\left[w_{-}\right]:=\{w \llbracket 1 \rrbracket-\operatorname{Min}[$ datacorrte $\llbracket 1 \rrbracket \llbracket 1 \rrbracket, 0], w \llbracket 2 \rrbracket\}$

datate $=$ Map [fnshift, datacorrte $]$;

This is a plot of the corrected radial wall expansion data. Specify the shot number for the label: ListPlot [datate, Frame $\rightarrow$ True, PlotLabel $\rightarrow$ "Shot\#15-2729 Corrected Film Data",
Framelabel $\rightarrow\{$ "Time ( $\mu$ sec) ", "Radial wall expansion (mm)" $\}]$

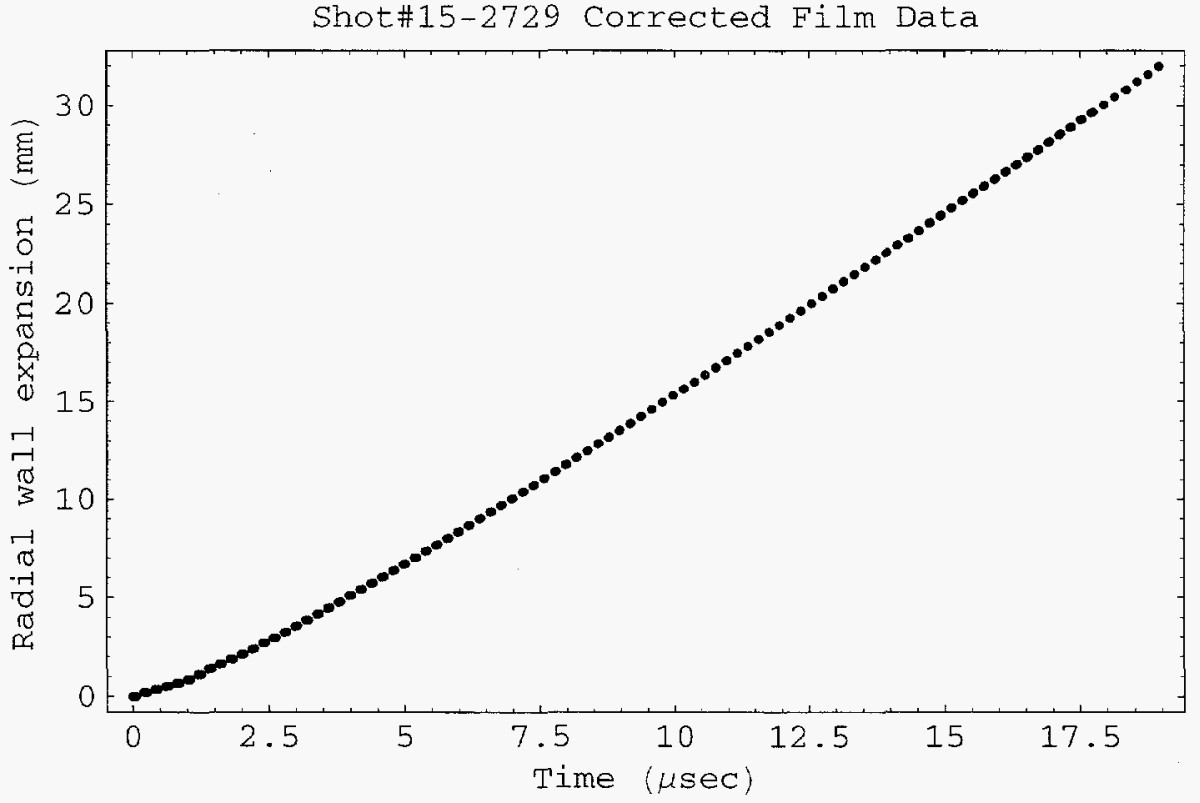

Delete the following intermediate plot after evaluation:

$$
\text { plot } 3 \text { = ListPlot [atate] }
$$

This set of commands is a routine for writing data to an external file.

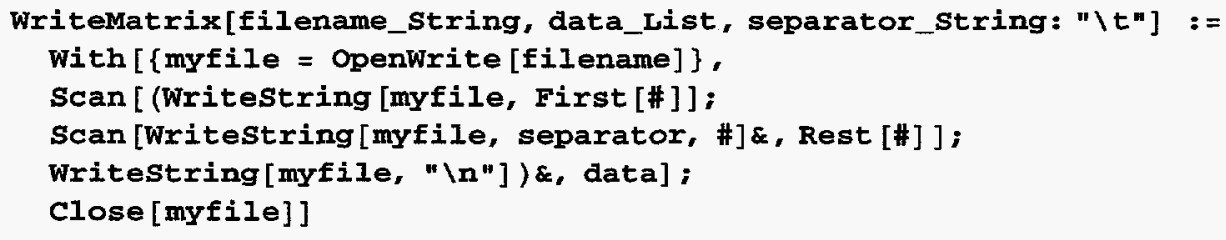

Write the final data set to a file, so that it may be used outside this notebook:

WriteMatrix["15-2729te.dat", datate]

$15-2729$ te. dat 


\section{Data Fitting}

Load the NonlinearF it package:

Needs[ "Statistics'NonlinearFit'"]

\section{- 6.1 Fitting Form}

Use the following rational polynomial fitting form, where vro is the asymptotic radial velocity, aro is the initial radial acceleration, to is a virtual fit origin, and $\omega$ is a fitting parameter:

$$
\begin{aligned}
& \text { efit }\left[t_{-}, \operatorname{vr} \infty_{-}, \operatorname{ar} 0_{-}, \omega_{-}, t 0_{-}\right]=\frac{\operatorname{vr\infty }(t-t 0)\left((1+(t-t 0))^{\omega}-1\right)}{\frac{2 \omega \operatorname{vr} \infty}{\operatorname{aro}}+\left((1+(t-t 0))^{\omega}-1\right)} \\
& \frac{\left(-1+(1+t-t 0)^{\omega}\right)(t-t 0) \operatorname{vr} \infty}{-1+(1+t-t 0)^{\omega}+\frac{2 \operatorname{vro\omega }}{\operatorname{aro}}}
\end{aligned}
$$

\section{- 6.2 First Pass}

It is necessary to first get an approximate fit using a reduced number of parameters; otherwise, the algorithm may not converge to the correct answer. A second pass will then fit all the parameters simultaneously.

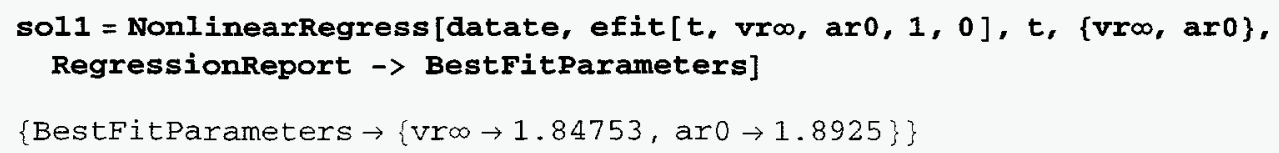

These commands assign the fitted parameters to variables:

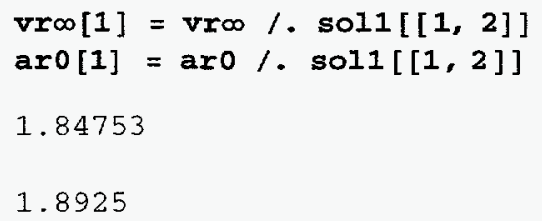

These commands compute the 10x fit residuals:

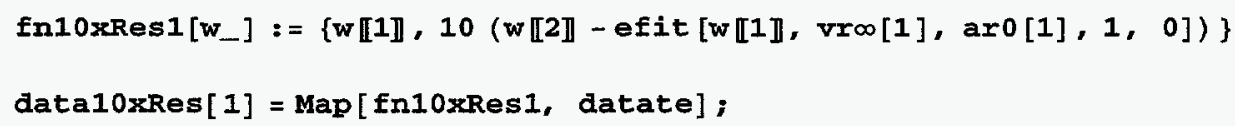

This is a plot of the first pass 10x fit residuals. Specify the shot number for the label: 


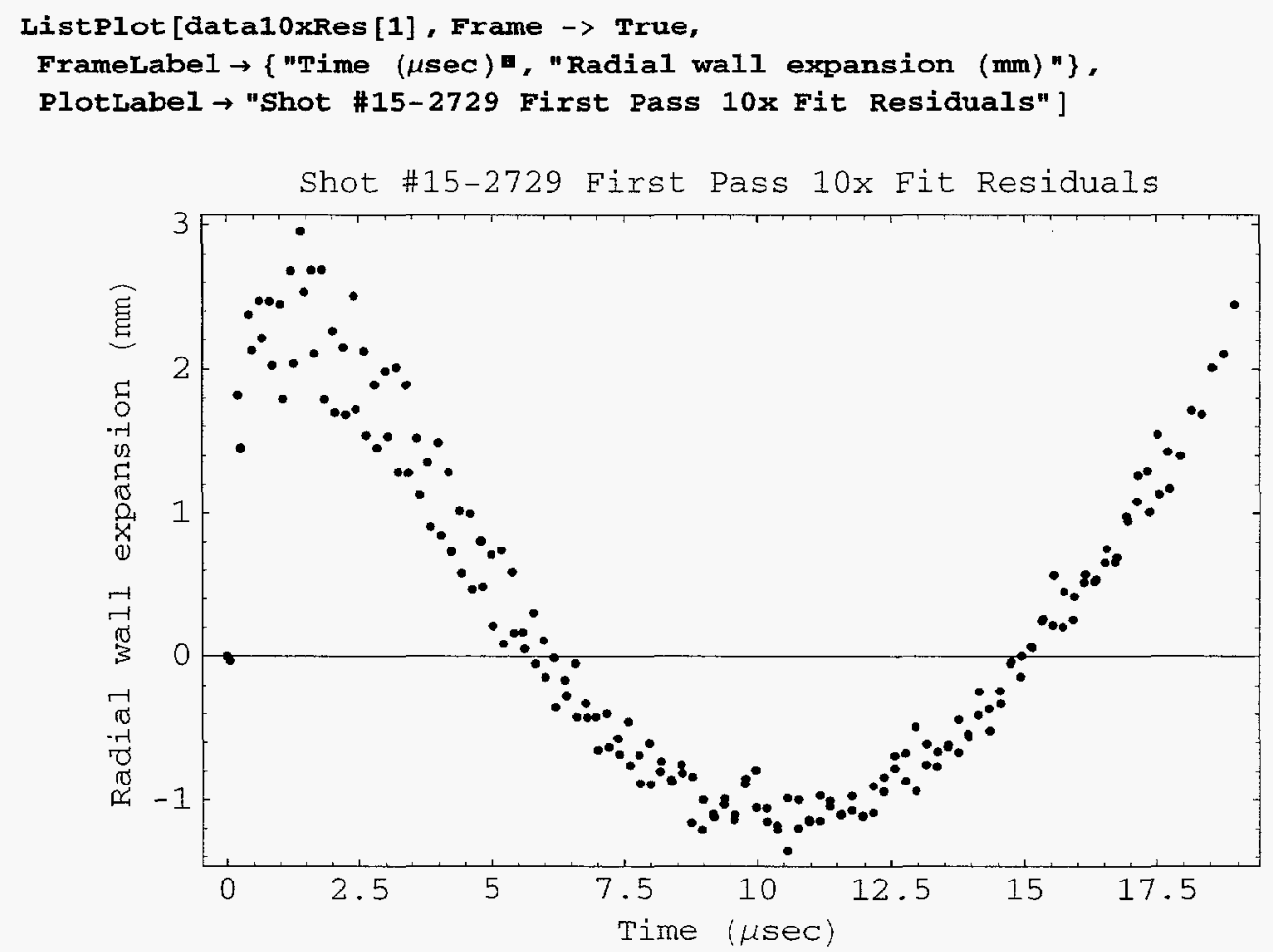

\section{- 6.3 SecondPass}

Now use the parameters from the first pass as starting values, and allow a finite virtual origin t0 and and arbitrary exponent $\omega$. The goal is for the fit residuals to oscillate symmetrically around zero. In some cases it may be desirable to chop off some of the initial data points when fitting, to give the fit the desired properties. In other cases the tube may break near the end of the record. In that case the tube appears to accelerate because of the smoke squirting out. If both sides of the tube are read, the record may also become asymmetric following break up. (A rule of thumb is that the tube usually expands to about three times its initial diameter before breaking.) If it is apparent that the tube is breaking, the associated data points should be truncated and the data should be re-fit.

\section{- 6.3.1 Truncated data set}

The parameter tfitmin is the time below which the data is to be dropped for fitting. Its value should be zero initially, and modified only if necessary to achieve the desired fit. Specify tfitmin :

tfitmin $=0$ (* nominal value zero *)

0

The parameter tfitmax is the time above which the data is to be dropped for fitting. Its value should be Tmax initially, and modified only if there is evidence that the tube broke before the end of the record. Specify tfitmax : 
tfitmax $=$ Tmax (* nominal value $T$ max *)

18.8924

These commands chop low and high parts seperately:

datatechopLo $=$ Drop $[$ datate, Floor [Length $[$ datate $] *$ tfitmin $/$ Tmax $]$ ];

datatechopHi $=\operatorname{Drop}[$ datate, Floor [Length [datate] * $($ Tmax - tfitmax $) / T \max ]]$;

The desired "chopped" file is the intersection of the above two lists:

datatechop = Intersection [ datatechopLo, datatechopHi] ;

\subsubsection{Final Fit}

This fit optimizes all the fitting parameters simultaneously, starting with reasonable guesses:

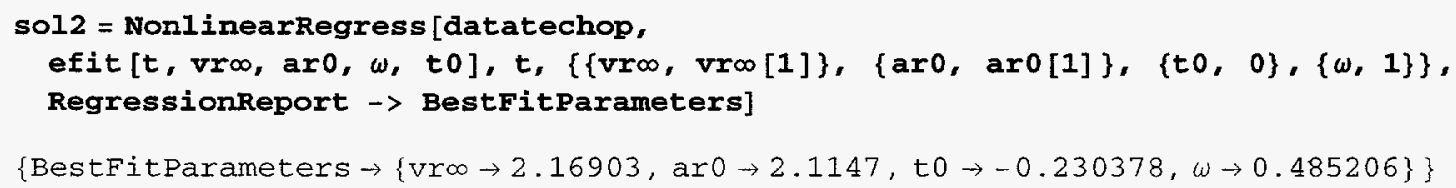

These commands assign the fitted parameters to variables:

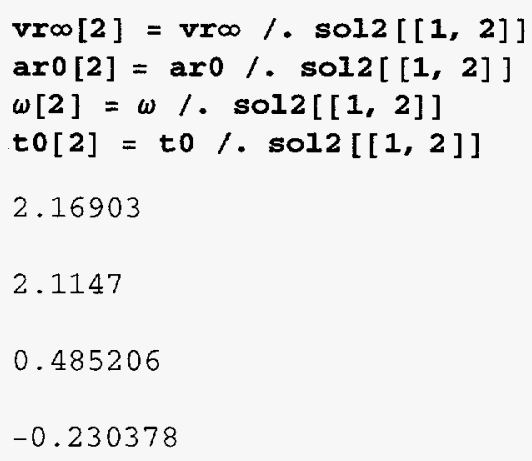

\section{- 6.3.3 Examination of fit}

Now compute the residuals of the second fit. Examine the residual pattern over the whole range, even if the fitting file was truncated:

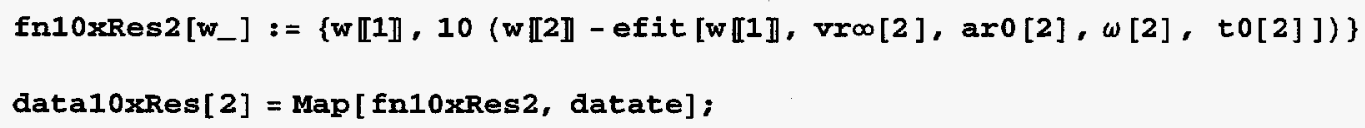

This is a plot of the second pass $10 x$ fit residuals. These should look significantly better than the first. Specify the shot number for the label: 


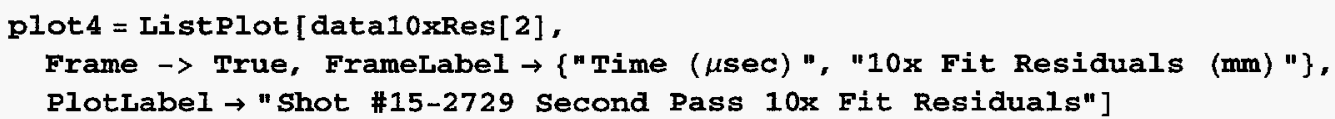

Shot \#15-2729 Second Pass 10x Fit Residuals

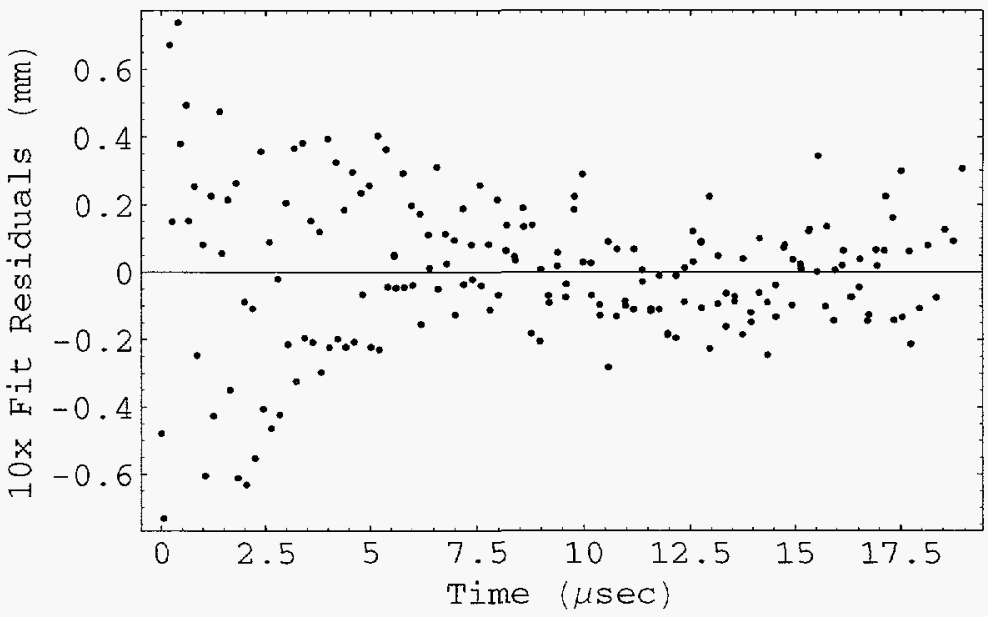

Delete the following intermediate plots after evaluation:

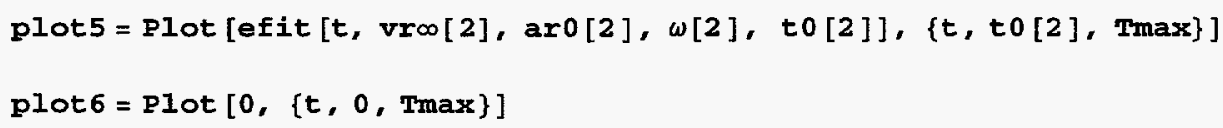

This is a plot of the corrected radial wall expansion data, fit, and 10x fit residuals. Specify the shot number for the label:

Show [plot3, plot4, plot5, plot6,

Frame $\rightarrow$ True, Prolog $\rightarrow$ TThickness $[0.002]$, Pointsize $[0.005]\}$,

Framelabel $\rightarrow$ \{"Time ( $\mu$ sec) ", "Radial wall expansion (mm)"\},

PlotLabel $\rightarrow$ "Shot \#15-2729 Film Data, Fit, and 10x Fit Residuals"]

Shot \#15-2729 Film Data, Fit, and 10x Fit Residuals

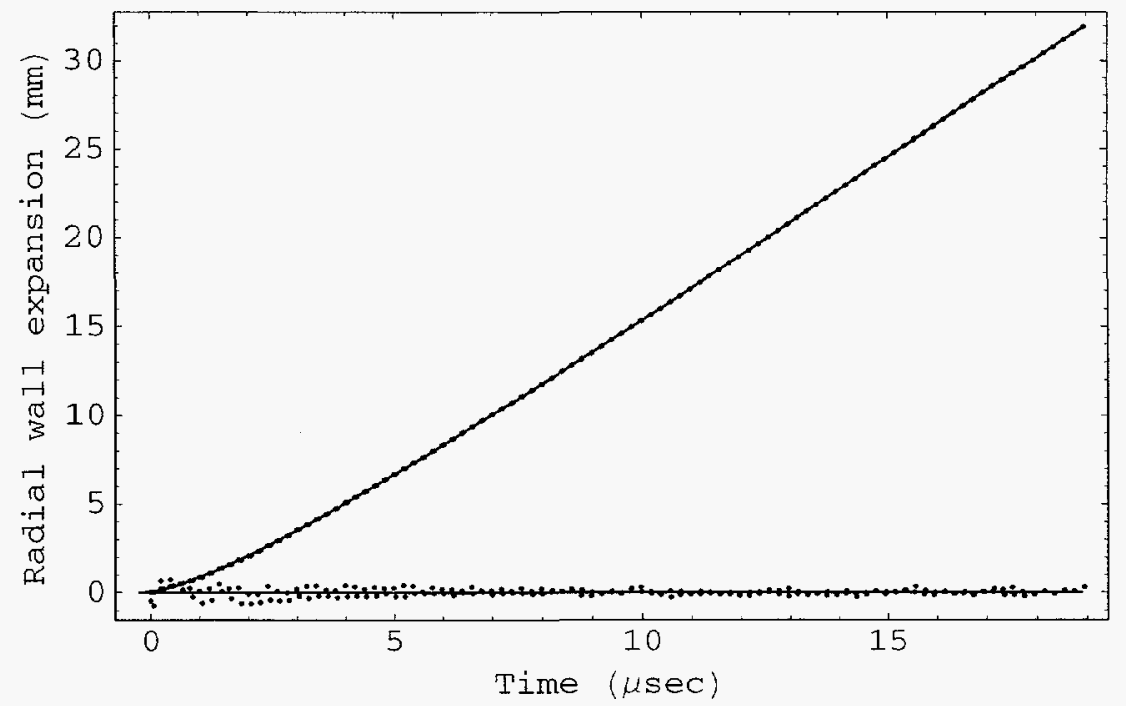




\section{- 6.3.4 Final Fit}

This is the formula used for subsequent evaluations, in which the virtual time origin is zeroed out. (The time origin is unimportant because the measures we shall compute are quoted as a function of tube expansion.)

$$
\begin{aligned}
& \operatorname{er}\left[t_{-}\right]=\operatorname{efit}[t, \operatorname{vr} \infty[2], \operatorname{aro}[2], \omega[2], 0] \\
& \frac{2.16903 t\left(-1+(1+t)^{0.485206}\right)}{-0.00465887+(1+t)^{0.485206}}
\end{aligned}
$$

\section{Radial Wall Velocity}

The radial wall velocity as a function of time is given by:

$\operatorname{vr}[t]=\operatorname{er}[t] ;$

(The radial velocity is not the same as the material velocity of the wall, but is close to it.) The following are radial velocities at the three standard expansions. The computed times correspond to the indicated expansion in millimeters. For example, " $t 6$ " is the time at which the wall achieved $6 \mathrm{~mm}$ radial expansion, and so on.

$t 6=t /$. FindRoot $[\operatorname{er}[t]==6,\{t, 1\}]$

4.8085

$\operatorname{vr}[t 6]$

1.61856

$t 19=t /$. FindRoot $[\operatorname{er}[t]==19,\{t, 1\}]$

12.2435

$\operatorname{vr}[t 19]$

1.82906

$t 30=t /$. FinaRoot $[\operatorname{er}[t]==30,\{t, 1\}]$

18.1482

$\operatorname{vr}[t 30]$

1.8906

Delete the following intermediate plot after evaluation:

plot7 = ParametricPlot $[\{\operatorname{er}[t], \operatorname{vr}[t]\},\{t, 0, t 30\}]$;

This plot shows the radial wall velocity as a parametric function of the radial wall expansion. Specify the shot number for the label: 
ParametricPlot $[\{\operatorname{er}[t], \operatorname{vr}[t]\},\{t, 0, t 30\}$, Frame $\rightarrow$ True, Prolog $\rightarrow$ Thickness $[0.002]$, Framelabel $\rightarrow$ \{"Radial wall expansion $(\mathrm{mm}) "$ " "Radial wall velocity (mm/ $\mu \mathrm{sec}$ )" $\}$, PlotLabel $\rightarrow$ "Shot \#15-2729 Radial Wall Velocity vs. Radial Wall Expansion"]

Shot \#15-2729 Radial Wall Velocity vs. Radial Wall Expansion

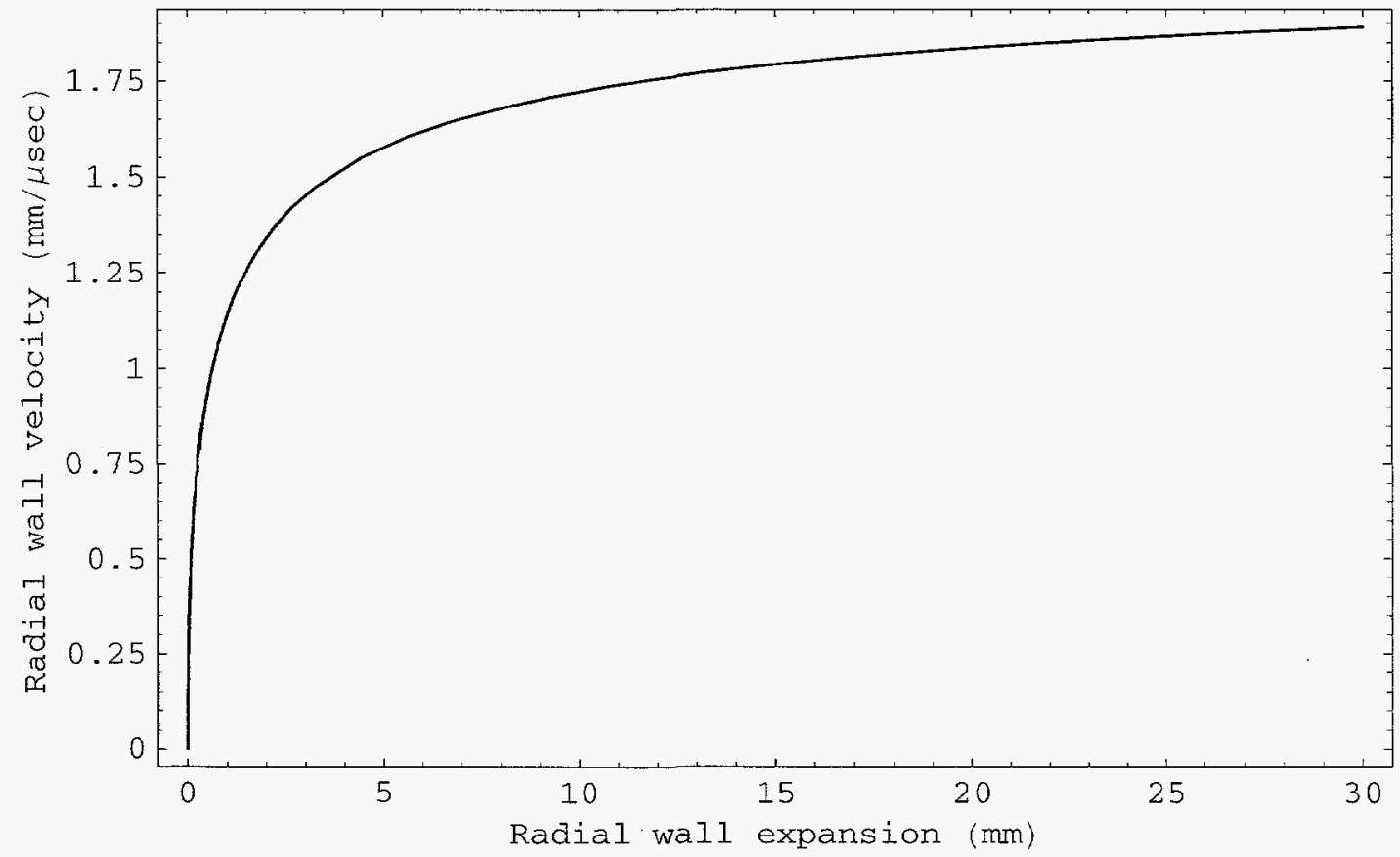

\section{Cylinder Energy}

The cylinder energy is defined as the specific kinetic energy of the wall based on the radial component of its velocity. It is not quite the same as the total specific kinetic energy of the wall, but it is close.

$$
\operatorname{Esp}\left[v r_{-}\right]=\frac{v r^{2}}{2}
$$

This plot shows the cylinder energy as a function of the radial wall expansion. Specify the shot number for the label: 


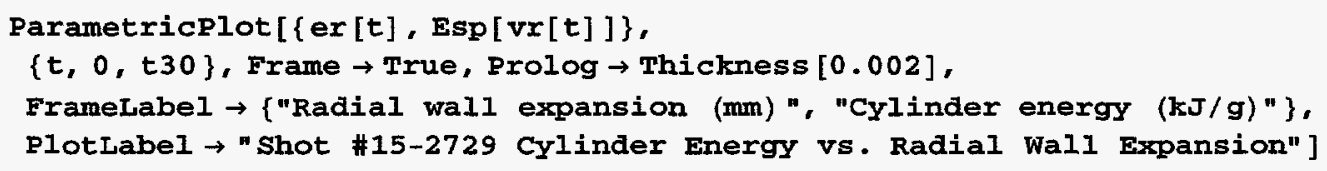

Shot \#15-2729 Cylinder Energy vs. Radial Wall Expansion

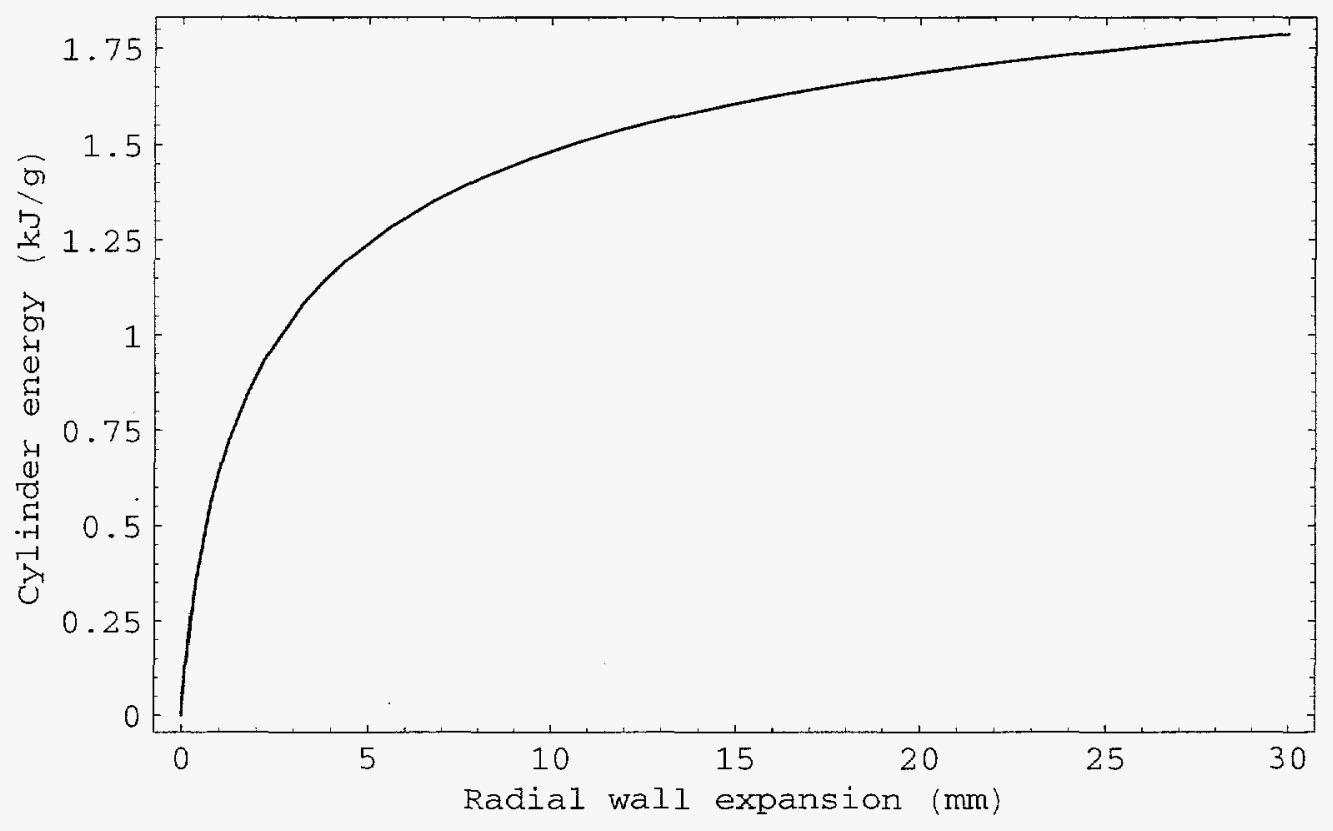

Compute the cylinder energy at the three standard radial expansions:

$\mathrm{E} 6=\operatorname{Esp}[\mathrm{Vr}[\mathrm{t6}]](* \mathrm{MJ} / \mathrm{kg} *)$

1.30987

The "standard" cylinder energy is computed at $19 \mathrm{~mm}$ radial expansion:

$\mathbf{E} 19=\operatorname{Esp}[\operatorname{vr}[t 19]](* \mathbf{M J} / \mathrm{kg} *)$

1.67273

$\mathbf{E} 30=\operatorname{Esp}[\operatorname{vr}[t 30]](* \mathbf{M J} / \mathbf{k g} *)$

1.78718

The cylinder energy may also be extrapolated to infinite expansion:

$\mathbf{E}_{\infty}=\operatorname{Esp}[\mathrm{Vr} \infty[2]] \quad(* \mathrm{MJ} / \mathbf{k g} *)$

2.35234 


\section{Gurney Energy}

The Gurney equation for cylindrical geometry is:

$$
\mathrm{G}\left[\mathrm{vr_{- }}\right]=\frac{\mathrm{vr} \mathrm{r}^{2}}{2}\left(\frac{1}{2}+\frac{\rho \mathrm{w}\left(\left(\frac{\mathrm{R} 0}{\mathrm{x} 0}\right)^{2}-1\right)}{\rho 0}\right)
$$

$1.32358 \mathrm{vr}^{2}$

This plot shows the Gurney energy as a function of the radial wall expansion. Specify the shot number for the label:
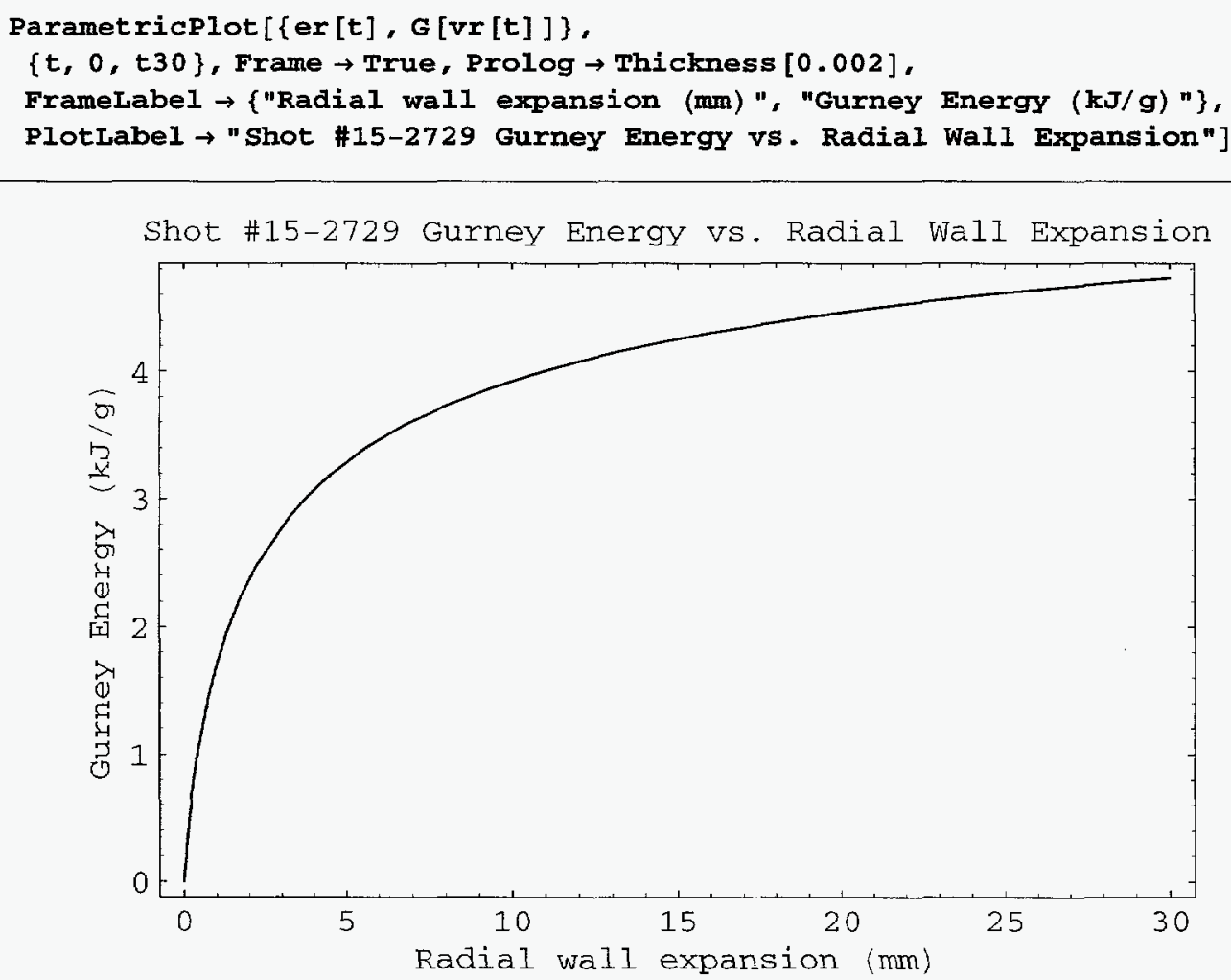

Compute the Gurney energy at the three standard radial expansions:

$$
\mathbf{G 6}=\mathbf{G}[\operatorname{Vr}[t 6]] \quad(* \mathbf{M J} / \mathbf{k g} *)
$$

3.46743

The "standard" Gurney energy is computed at $19 \mathrm{~mm}$ radial expansion:

$$
\text { G19 }=G[\operatorname{vr}[\mathrm{t} 19]] \quad(* \mathrm{MJ} / \mathrm{kg} *)
$$

4.42799

The "asymptotic" Gurney energy, evaluated at the asymptotic expansion velocity, is: 
$\mathrm{G}_{\infty}=\mathrm{G}[\mathrm{vr} \infty[2]]$ (* MJ/kg $*$ )

6.22701

\section{Axial Velocity}

Specify the pin data in $(t, z)$ pairs, with $t$ in microseconds and $z$ in millimeters:

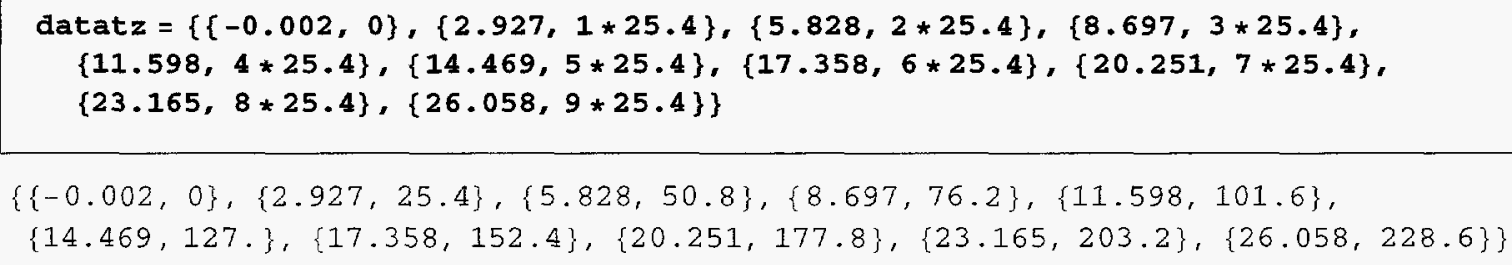

Delete the following intermediate plot after evaluation:

$$
\text { plot } 8=\text { Listplot }[\text { datatz }]
$$

This command performs a linear fit to the data. If there are any bad pins, eliminate them from the fit using the Drop command):

$$
\begin{aligned}
& z\left[t_{-}\right]=\operatorname{Fit}[\text { datatz, }\{1, t\}, t] \\
& -0.184422+8.78292 t
\end{aligned}
$$

The maximum pin time is:

tmaxpins = datatz $[$ Length $[$ datatz $] \rrbracket \llbracket 1 \rrbracket$

26.058

These commands compute the $10 x$ fit residuals :

$$
\begin{aligned}
& \text { fn10xRes } \left.3\left[w_{-}\right]:=\{w \llbracket 1], 10(w \llbracket 2 \rrbracket-z[w \llbracket 1 \rrbracket])\right\} \\
& \text { data10xRes[3] = Map [fn10xRes3, datatz] ; }
\end{aligned}
$$

Delete the following intermediate plots after evaluation:

$$
\begin{aligned}
& \text { plot9 }=\text { Plot }[z[t],\{t, 0, \text { tmaxpins }\}] \\
& \text { plot } 10=\text { ListPlot }[\text { data10xRes }[3]] \\
& \text { plot } 11=\operatorname{Plot}[0,\{t, 0, \text { tmaxpins }\}]
\end{aligned}
$$

This plot shows the $t-z$ pin data, the linear fit, and the $10 x$ fit residuals. Specify the shot number for the label: 


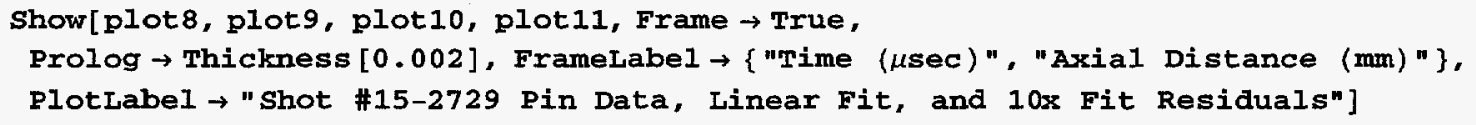

Shot \#15-2729 Pin Data, Linear Fit, and 10x Fit Residuals

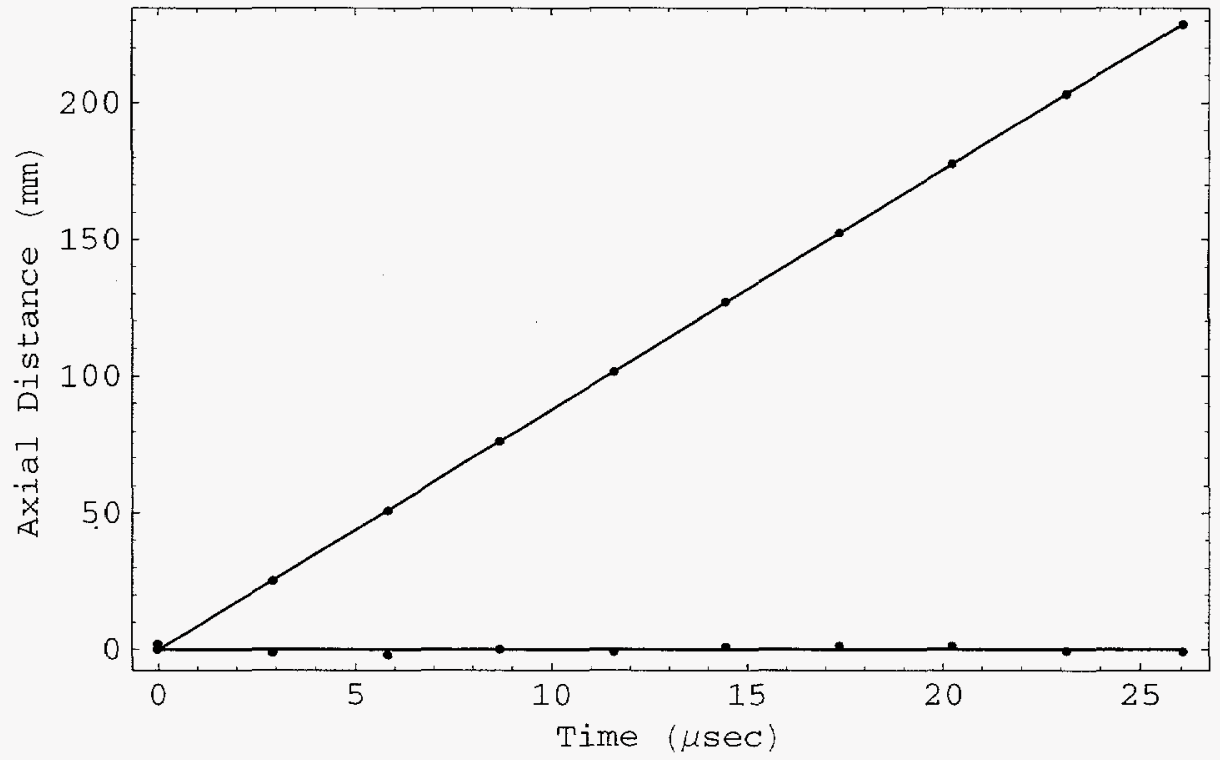

The detonation velocity is the slope of the above plot:

Do = Coefficient $[z[t], t]$

8.78292

Perform a linear regression analysis:

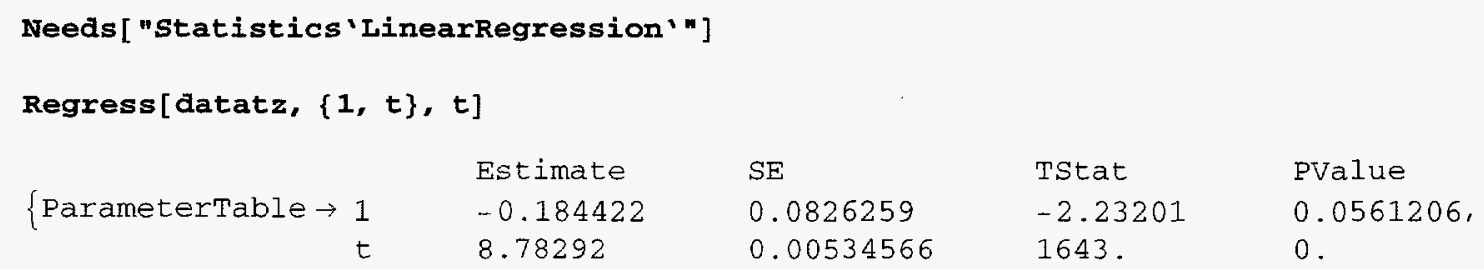

